\title{
A STRONG GENERALIZATION OF HELGASON'S THEOREM
}

\author{
KENNETH D. JOHNSON
}

\begin{abstract}
Let $G$ be a simple Lie group with $K A N$ an Iwasawa decomposition of $G$, and let $M$ be the centralizer of $A$ in $K$. Suppose $K_{1}$ is a fixed, closed, normal, analytic subgroup of $K$, and set $\mathbf{P}\left(K_{1}\right)$ equal to the set of all parabolic subgroups $P$ of $G$ which contain $M A N$ such that $K_{1} P=G$ and $K_{1} \cap P$ is normal in the reductive part of $P$. Suppose $\pi: G \rightarrow G L(V)$ is an irreducible representation of $G$. Then, if $\mathbf{P}\left(K_{1}\right) \neq \varnothing$, we obtain necessary and sufficient conditions for $V^{K_{1}}$, the space of $K_{1}$-fixed vectors, to be $\neq(0)$. Moreover, reciprocity formulas are obtained which determine $\operatorname{dim} V^{K_{1}}$.
\end{abstract}

1. Introduction. Let $G$ be a connected, real, simple, noncompact Lie group with finite center, and $K$ a maximal compact subgroup. If $g$ is the Lie algebra of $G$ and $\mathfrak{f}$ the Lie algebra of $K, \mathfrak{g}$ has Cartan decomposition, $\mathfrak{g}=\mathfrak{f}+\mathfrak{p}$ where $\mathfrak{p}$ is orthogonal to $\mathfrak{f}$ with respect to the Killing form. Fix $\mathfrak{a}$, a maximal abelian subalgebra of $\mathfrak{p}$, and set $A=\exp$ a. Let $M$ be the centralizer of $A$ in $K$ and denote its Lie algebra by $\mathfrak{m}$.

If $\mathfrak{a}^{*}$ is the real dual space of $\mathfrak{a}$ and $\lambda \in \mathfrak{a}^{*} \sim\{0\}$, set

$$
\mathfrak{g}_{\lambda}=\{X \in \mathfrak{g}:[H X]=\lambda(H) X \text { for all } H \in \mathfrak{a}\},
$$

and call $\lambda$ a restricted root if $\mathfrak{g}_{\lambda} \neq(0)$. Denote the set of restricted roots by $\Sigma_{0}$ and the set of points in $a$ where no restricted root vanishes by $a^{\prime}$. Let $\mathfrak{a}^{+}$be a connected component of $\mathfrak{a}^{\prime}$, and let $\Sigma_{0}^{+}$be the set of restricted roots which are positive on $\mathfrak{a}^{+}$. Now $\Sigma_{0}=\Sigma_{0}^{+} \cup\left(-\Sigma_{0}^{+}\right)$, and if

$$
\mathfrak{n}=\left\langle\mathfrak{g}_{\lambda}: \lambda \in \Sigma_{0}^{+}\right\rangle, \quad \overline{\mathfrak{n}}=\left\langle\mathrm{g}_{-\lambda}: \lambda \in \Sigma_{0}^{+}\right\rangle, \quad \mathfrak{g}=\overline{\mathfrak{n}}+\mathfrak{m}+\mathfrak{a}+\mathfrak{n} .
$$

If $N=\exp \mathrm{n}, G=K A N$ is the Iwasawa decomposition of $G$.

We assume throughout this paper that $G$ is contained in a complex, simple, connected, simply connected Lie group $G_{\mathbf{C}}$ whose Lie algebras $g_{\mathbf{C}}$ is the complexification of $\mathfrak{g}$. The restriction map yields a one-to-one correspondence between irreducible holomorphic representations of $G_{\mathbf{C}}$ and finite dimensional, complex, irreducible representations of $G$. Suppose now that $\pi: G \rightarrow G L(V)$ is a finite dimensional, complex, irreducible representation. A well-known result of Helgason [H2] states that $\operatorname{dim} V^{K}=\operatorname{dim} V^{M N}$, with $\operatorname{dim} V^{K}=0$ or 1 , where for any subset $U$ of $G, V^{U}$ is the space of $U$-fixed vectors. Moreover, Helgason also obtained a characterization of the representations $\pi$ for which $V^{K} \neq(0)$. Helgason's theorem was first extended (in a strict sense) by H. Schlichtkrull [S].

Received by the editors May 22, 1986.

1980 Mathematics Subject Classification (1985 Revision). Primary 22E46; Secondary 57S20, 22E47.

Key words and phrases. Reciprocity pair, parabolic group, representation.

This work was partially supported by a grant from the National Science Foundation. 
Schlichtkrull considered groups $G$ for which $K$ has a one dimensional center. That is, groups $G$ for which $G / K$ is an irreducible Hermitian symmetric space. If $K_{1}=[K, K]$, the commutator subgroup of $K$, Schlichtkrull characterized the representations $\pi$ for which $V^{K_{1}} \neq(0)$, and showed that $V^{K_{1}} \neq(0)$ if and only if $V^{M_{1} N} \neq(0)$ where $K_{1} \cap M=M_{1}$.

In a similar vein but in an apparently unrelated work [G], S. Gelbart considered the group $S O(n)$. Suppose $[n / 2]<q \leqslant n$, and $S O(q)$ is realized as a subgroup of $S O(n)$ in standard form. Gelbart characterized the irreducible representations $\pi$ : $S O(n) \rightarrow G L(V)$ for which $V^{S O(q)} \neq(0)$, and by an "act of providence" obtained an interesting reciprocity formula showing that $\operatorname{dim} V^{S O(q)}$ is in fact equal to the dimension of certain (depending on $\pi$ ) irreducible representation of $U(n-2 q)$ ). In [G-K], K. Gross and R. Kunze succeeded in obtaining a strong generalization of Gelbart's result. Using their concept of bitriangular system on complex groups, Gross and Kunze were able to establish results analogous to Gelbart's for all classical groups.

Our main goal here is to provide a unified treatment of these results. Rather than using the concept of bitriangular systems of Gross and Kunze, we shall use an approach which is somewhat more in keeping with the original approach of Helgason. In fact, Helgason's theorem will be a basic stepping stone toward our main result. Although our approach lacks some of the algebraic elegance of Gross and Kunze, our method does provides a certain completeness. We also trust that $\S 3$ will be of interest to persons studying real homogeneous varieties.

Suppose $\mathbf{P}$ is the set of all parabolic subgroups of $G$ which contain $M A N$. If $k_{1}$ is an ideal in $k$, let $K_{1}$ be the analytic subgroup of $K$ with Lie algebra $k_{1}$.

Definition 1.1. Let $\mathbf{P}\left(K_{1}\right)$ be the set of all $\tilde{P} \in \mathbf{P}$ :

i. $K_{1} \tilde{P}=G$; and

ii. $K_{1} \cap \tilde{P}=M_{1}$ is normal in the reductive part of $\tilde{P}$.

For $\tilde{P} \in \mathbf{P}\left(K_{1}\right)$ we call $\left(K_{1}, \tilde{P}\right)$ a reciprocity pair in $G$.

In $\S \S 5,6$ and 7 , we shall see that if $\mathbf{P}\left(K_{1}\right)$ is nonempty $\left|P\left(K_{1}\right)\right|=1$ unless $G=\operatorname{Spin}_{0}(n, n)$ and $K_{1}=\operatorname{Spin}(n)$. In this case, $\left|P\left(K_{1}\right)\right|=2$.

Suppose $K_{1}$ is taken as above and $\mathbf{P}\left(K_{1}\right)$ is nonempty. For $\tilde{P} \in \mathbf{P}\left(K_{1}\right)$ suppose $\tilde{P}$ has Langlands decomposition $\tilde{M} \tilde{A} \tilde{N}$. We now state our main result.

Theorem A. Let $\pi: G \rightarrow G L(V)$ be a finite dimensional, complex, irreducible representation of $G$. If either $\left(G, K_{1}\right) \neq(S U(n, n), U(n))$, or if $\left(G, K_{1}\right)=$ $(S U(n, n), U(n))$ and $\operatorname{dim} V^{K_{1}} \neq 0$,

$$
\operatorname{dim} V^{K_{1}}=\min \operatorname{dim}\left\{V^{\left(K_{1} \cap \tilde{M}\right) \tilde{N}}: \tilde{P} \in \mathbf{P}\left(K_{1}\right)\right\} .
$$

Our proof of this theorem will proceed as follows. After first developing some algebraic machinery and recalling properties of roots in $\S 2$, we describe the group $M$ in $\S 3$. In particular, we determine the number of components of $M$. In $\S 4$, we examine some results in Lie algebra cohomology which will be needed in $\S 5$. In $\S \S 5$, 6 and 7 we classify all reciprocity pairs in $G$ for different $K_{1}$. We obtain some results in $\S 8$ concerning representations of $G$ which enable us to prove Theorem A in $\S 9$.

Finally, I would like to express my appreciation to Ray Kunze and Henrik Schlichtkrull for many useful discussions. 
2. Algebraic preliminaries. If $t$ is a Cartan subalgebra of $m, t+a$ is a Cartan subalgebra of $\mathfrak{g}$, and $t_{\mathbf{c}}+a_{\mathbf{C}}$ is a Cartan subalgebra of $\mathfrak{g}_{\mathbf{C}}$. Set $\mathfrak{h}=\sqrt{-1} \mathfrak{t}+\mathfrak{a}$, and let $\Sigma \subset \mathfrak{h}^{*}$ be the root system of $\mathfrak{g}_{\mathbf{C}}$ with respect to $\mathfrak{h}$. For $\alpha \in \Sigma$ let $\mathfrak{g}_{\mathbf{C}, \alpha}$ denote the corresponding root space. If $r: \mathfrak{h}^{*} \rightarrow \mathfrak{a}^{*}$ is the restriction map,

$$
\Sigma_{0}=r(\Sigma) \sim\{0\} \text {. }
$$

Moreover $r(\alpha)=0$ if and only if $\mathrm{g}_{\mathbf{C}, \alpha} \subset \mathfrak{m}_{\mathbf{C}}$. Set

$$
\Sigma_{00}=\{\alpha \in \Sigma: r(\alpha)=0\} \text {, }
$$

and define

$$
(\sqrt{-1} \mathrm{t})^{\prime}=\left\{H \in \sqrt{-1} \mathrm{t}: \alpha(H) \neq 0 \text { for all } \alpha \in \Sigma_{00}\right\} .
$$

Fix $(\sqrt{-1} \mathrm{t})^{+}$a connected component of $(\sqrt{-1} \mathrm{t})^{\prime}$, and let

$$
\Sigma_{00}^{+}=\left\{\alpha \in \Sigma_{00}: \alpha(H)>0 \text { for all } H \in(\sqrt{-1} \mathrm{t})^{+}\right\} .
$$

This allows us to define an ordering on $\Sigma$ compatible with the ordering on $\Sigma_{0}$ by setting

$$
\Sigma^{+}=\left\{\alpha: r(\alpha) \in \Sigma_{0}^{+}\right\} \cup \Sigma_{00}^{+} .
$$

This procedure yields $|W(\mathfrak{m}, \mathfrak{t})|$ compatible orderings on $\Sigma$ where $W(\mathfrak{m}, \mathfrak{t})$ is the Weyl group of $\mathfrak{m}$.

Let $\Phi \subset \Sigma^{+}, \Phi_{0} \subset \Sigma_{0}^{+}$and $\Phi_{00} \subset \Sigma_{00}^{+}$be the respective simple root systems of $\Sigma$, $\Sigma_{0}$ and $\Sigma_{00}$. Recall that the Satake diagram of $g$ is obtained from the Dynkin diagram of $\mathrm{g}_{\mathbf{C}}$ as follows:

1. Blacken $\alpha \in \Phi$ if $\alpha \in \Phi_{00}$; and,

2. If $\alpha, \beta \in \Phi \sim \Phi_{00}$, and $r(\alpha)=r(\beta)$, join $\alpha$ to $\beta$ with a double headed arrow. (This can occur only when all roots of $\Sigma$ are of equal length.) Recall that for $\lambda \in \Phi_{0}$ there are at most 2 roots in $\Phi$ whose image under $r$ is $\lambda$.

Suppose $\langle$,$\rangle is the inner product on \mathfrak{h}^{*}$ dual to the restriction of the Killing form on $\mathfrak{h}$, and for $\mu \in \mathfrak{h}^{*}$ let $H_{\mu}$ be the element of $\mathfrak{h}$ for which $B\left(H_{\mu}, H\right)=\mu(H)$ for all $H \in \mathfrak{h}$. If $\bar{L}$ is the lattice generated by the set $\left\{2 H_{\lambda} /\langle\lambda, \lambda\rangle: \lambda \in \Sigma_{0}\right\}$, it is well known [L, p. 77] that the group $\bar{\Gamma}=(\exp i \mathrm{a}) \cap G$ is in fact $\exp i \pi \bar{L}$. If

$$
L=\left\{H \in \mathfrak{a}: \lambda(H) \in \mathbf{Z} \text { for all } \lambda \in \Sigma_{0}\right\},
$$

$\bar{L}$ is a sublattice of $L$, and hence, if $\Gamma=\exp \pi i L, \bar{\Gamma}$ is a subgroup of $\Gamma$.

Setting

$$
\tilde{G}=(\text { Aut } g) \cap \operatorname{Int} g_{C},
$$

we have

$$
\tilde{G}=\operatorname{Ad} \Gamma \cdot \operatorname{Int} g=\operatorname{Ad} \Gamma \cdot \operatorname{Ad} G,
$$

and

$$
[\tilde{G}: \operatorname{Ad} G]=[\Gamma: \bar{\Gamma}]=[L: \bar{L}] .
$$

(This follows from Theorem 7.2, Chapter 3 of [H1] and Proposition 1 of [K-R].) 
For $H \in \overline{\mathfrak{a}^{+}}$, set

$$
\begin{aligned}
\mathfrak{n}(H) & =\left\langle\mathfrak{g}_{\lambda}: \lambda \in \Sigma_{0}^{+} \text {and } \lambda(H)>0\right\rangle, \\
\mathfrak{z}(H) & =\{X \in \mathfrak{g}:[H, X]=0\}, \\
\mathfrak{m}(H) & =[z(H), \mathfrak{z}(H)]+\mathfrak{z}(H) \cap \mathfrak{f}, \\
\mathfrak{a}(H) & =\mathfrak{z}(z(H)) \cap \mathfrak{a} .
\end{aligned}
$$

Then $z(H)=\mathfrak{m}(H)+\mathfrak{a}(H)$, and the algebra $\mathfrak{p}(H)=z(H)+\mathfrak{n}(H)$ is a parabolic subalgebra, and any parabolic subalgebra containing $m+a+\mathfrak{n}$ is of this form. Set $A(H)=\exp \mathfrak{a}(H), N(H)=\exp \mathfrak{n}(H)$, and if

$$
Z(H)=\{g \in G: \operatorname{Ad} g(H)=H\},
$$

set

$$
M(H)=[Z(H), Z(H)] \cdot Z(H) \cap K .
$$

Then $P(H)=M(H) A(H) N(H)$ is the parabolic subgroup of $G$ with Lie algebra $\mathfrak{p}(H)$ written in its Langlands decomposition. Moreover, any element of $\mathbf{P}$ is of this form. Now $M(H) A(H)=Z(H)$ is the reductive part of $P(H)$ and $N(H)$ is its unipotent radical. If $M_{0}(H)$ is the connected component of the identity of $M(H)$,

$$
P_{0}(H)=M_{0}(H) A(H) N(H)
$$

is the connected component of the identity of $P(H)$ and

$$
P(H)=\bar{\Gamma} \cdot P_{0}(H) \text {. }
$$

Suppose $\left\{H_{\alpha}^{0}: \alpha \in \Phi_{0}\right\} \subset \mathfrak{a}$ is the dual basis to $\Phi_{0}$. That is, $\alpha\left(H_{\beta}^{0}\right)=0$ if $\beta \neq \alpha$ and $\alpha\left(H_{\alpha}^{0}\right)=1$. For $I \subset \Phi_{0}$ fix

$$
H_{I}^{0}=\sum_{\alpha \in I} H_{\alpha}^{0} .
$$

Set $P(I)=P\left(H_{I}^{0}\right), M(I)=M\left(H_{I}^{0}\right), A(I)=A\left(H_{I}^{0}\right)$, and $N(I)=N\left(H_{I}^{0}\right)$. Then if $H \in \overline{\mathrm{a}^{+}}, P(H)=P(I)$ where

$$
I=\left\{\alpha \in \Phi_{0}: \alpha(H)>0\right\} .
$$

Observe that $P(\phi)=G, P\left(\Phi_{0}\right)=B=M A N$, and if $I \subset J, P(J) \subset P(I)$.

Proposition 2.1. For $I \subset \Phi_{0}, Z(I)=M(I) A(I)$ is the group generated by $M(I) \cap K$ and $A$.

Proof. If $U$ is the group generated by $M(I) \cap K$ and $A$, clearly $U \subset Z(I)$. As $\bar{\Gamma} \subset U$, it suffices to show that $U$ and $Z(I)$ have the same Lie algebra.

If $X_{\lambda} \in \mathfrak{g}_{\lambda} \subset z(I)$,

$$
X_{\lambda}+\theta X_{\lambda} \in \mathfrak{m}(I) \cap \mathfrak{f} \subset \mathcal{u} .
$$

Moreover, there is an $H \in \mathfrak{a}$ for which $\lambda(H) \neq 0$. Thus

$$
\left[H, X_{\lambda}+\theta X_{\lambda}\right]=\alpha(H)\left(X_{\lambda}-\theta X_{\lambda}\right) \in \mathfrak{u} .
$$

Hence $X_{\lambda} \in \mathfrak{u}, g_{\lambda} \subset \mathfrak{u}$ and as $\mathfrak{m}+\mathfrak{a} \subset \mathfrak{u}$, we are done.

Proposition 2.2. Let $U$ be a subgroup of $K$. If $U \cap P(I)$ is a normal subgroup of $M(I), U \cap P(I)=U \cap M$. 
Proof. $u \in U \cap P(I)$ and $H \in \mathfrak{a}$, consider the curve $t \rightarrow x(t)$ in $U \cap M(I)$ given by

$$
t \rightarrow(\exp -t H) u(\exp t H) u^{-1}=x(t)
$$

Now

$$
x^{\prime}(0)=\operatorname{Ad} u(H)-H \in \mathfrak{u} \subset \mathfrak{k},
$$

and as $u \in U, x^{\prime}(0) \in \mathfrak{p}$. Thus $x^{\prime}(0)=0$ and so $u \in M$.

3. The group $M$. As $M=\bar{\Gamma} \cdot M_{0}$, we wish to first determine $\bar{\Gamma} \cap M_{0}$. From the definition of $\bar{\Gamma}$ we have that $\gamma^{2}=e$ for any $\gamma \in \bar{\Gamma}$. To get a better description of $\bar{\Gamma}$ in terms of generators set

$$
\Sigma_{0}^{\prime}=\left\{\alpha \in \Sigma_{0}: \frac{1}{2} \alpha \notin \Sigma_{0}\right\},
$$

and

$$
\Sigma_{0}^{\prime \prime}=\left\{\alpha \in \Sigma_{0}: 2 \alpha \notin \Sigma_{0}\right\} .
$$

Then $\Sigma_{0}^{\prime}$ and $\Sigma_{0}^{\prime \prime}$ are root systems of simple groups with $\Sigma_{0}=\Sigma_{0}^{\prime} \cup \Sigma_{0}^{\prime \prime}$. Now $\Phi_{0}$ is a set of simple roots of $\Sigma_{0}^{\prime}$, and $\Psi_{0}=\left(\Phi_{0} \cup 2 \Phi_{0}\right) \cap \Sigma_{0}^{\prime \prime}$ is a set of simple roots of $\Sigma_{0}^{\prime \prime}$. For $\alpha \in \Sigma_{0}$, set

$$
\gamma_{\alpha}=\exp 2 \pi i H_{\alpha} /\langle\alpha, \alpha\rangle .
$$

From [St, p. 2], it follows that any $H_{\alpha} /\langle\alpha, \alpha\rangle$ is an integral linear combination of $H_{\lambda} /\langle\lambda, \lambda\rangle$ where $\lambda \in \Phi_{0} \cup \Psi_{0}$. Hence

$$
\bar{\Gamma}=\left\langle\gamma_{\alpha}: \alpha \in \Phi_{0} \cup \Psi_{0}\right\rangle \text {. }
$$

That is, $\bar{\Gamma}$ is the group generated by $\left\{\gamma_{\alpha}: \alpha \in \Phi_{0} \cup \Psi_{0}\right\}$.

Proposition 3.1. $\bar{\Gamma}=\left\langle\gamma_{\alpha}: \alpha \in \Psi_{0}\right\rangle$.

Proof. It suffices to prove that if $\{\alpha, 2 \alpha\} \subset \Sigma_{0}, \gamma_{\alpha}=e$. However, as

$$
H_{\alpha} /\langle\alpha, \alpha\rangle=2 H_{2 \alpha} /\langle 2 \alpha, 2 \alpha\rangle \text {, }
$$

$\gamma_{\alpha}=\gamma_{2 \alpha}^{2}=e$.

Lemma 3.2. Suppose $r(\alpha)=\lambda$, and $H_{\alpha}+\theta H_{\alpha} \neq 0$. Then $\gamma_{\lambda} \in M_{0}$.

Proof. As $H_{\alpha}+\theta H_{\alpha} \neq 0$,

$$
\gamma_{\lambda}=\exp 2 \pi i H_{\alpha} /\langle\lambda, \lambda\rangle \cdot \nabla
$$

where

$$
\nabla=\exp -\pi i\left(H_{\alpha}+\theta H_{\alpha}\right) /\langle\lambda, \lambda\rangle \in M_{0} .
$$

Recall [L, p. 38] that for any $\beta \in \Sigma$,

$$
\exp 4 \pi i H_{\beta} /\langle\beta, \beta\rangle=e .
$$

Moreover, from [H2, p. 77] we have $\langle\alpha, \alpha\rangle=k\langle\lambda, \lambda\rangle$, with $k=1,2$ or 4 , and $k=1$ if and only if $\alpha+\theta \alpha=0$. Hence

$$
\exp 2 \pi i H_{\alpha} /\langle\lambda, \lambda\rangle=\exp 2 k \pi i H_{\alpha} /\langle\alpha, \alpha\rangle=e .
$$

Thus $\gamma_{\lambda} \in M_{0}$. 
Lemma 3.3. If $\{\lambda, 2 \lambda\} \subset \Sigma_{0}, \gamma_{2 \lambda} \in M_{0}$.

Proof. If $M^{\prime}$ is the normalizer of $M$ in $K, M^{\prime} / M=W(G, K)$ is the restricted Weyl group of $G$. Observe that $M^{\prime}$ normalizes $M_{0}$. If $\lambda, \lambda^{\prime} \in \Sigma_{0}$ and $\|\lambda\|=\left\|\lambda^{\prime}\right\|$, there is an $s \in W(G, K)$ such that $s \lambda=\lambda^{\prime}$. Hence it suffices to assume that $\lambda \in \Phi_{0}$.

Assume first that all roots in $\Sigma$ are of the same length and $r(\alpha)=\lambda$. Then as $2 \lambda \in \Sigma_{0}$ we have that $\alpha-\theta \alpha=2 \lambda \in \Sigma$ and $\alpha+\theta \alpha \neq 0$. Since

$$
\gamma_{2 \lambda}=\exp 2 \pi i H_{2 \lambda} /\langle 2 \lambda, 2 \lambda\rangle \text {, }
$$

and

$$
i H_{2 \lambda} /\langle 2 \lambda, 2 \lambda\rangle=i\left(H_{\alpha}-\theta H_{\alpha}\right) /\langle\alpha, \alpha\rangle=2 i H_{\alpha} /\langle\alpha, \alpha\rangle \bmod m,
$$

then $\gamma_{2 \lambda} \in M_{0}$.

As $\{\lambda, 2 \lambda\} \not \subset \Sigma_{0}$, if $G$ is a complex group or a real split group, to complete the proof it suffices from [H1, p. 354], to consider $G$ a nonsplit real form of $B_{l}, C_{l}$ or $F_{4}$. If $G$ is a real form of type $B_{l}, G=\operatorname{Spin}_{0}(p, q)$ and $\{\lambda, 2 \lambda\} \not \subset \Sigma_{0}$. Hence $G$ is either $\mathrm{Sp}(p, q)(p<q)$ or $F_{4(-20)}$. In both these cases if $r(\alpha)=\lambda$, we have that $\alpha,-\theta \alpha$ and $2 \lambda$ are short roots of $\Sigma$. Hence

$$
i H_{2 \lambda} /\langle 2 \lambda, 2 \lambda\rangle=i\left(H_{\alpha}-\theta H_{\alpha}\right) /\langle\alpha, \alpha\rangle=2 i H_{\alpha} /\langle\alpha, \alpha\rangle \bmod \mathrm{m},
$$

and this proves our result.

Let $R(\Phi)=\{\lambda \in \Phi: \lambda+\theta \lambda=0\}$, and identify $R(\Phi)$ with $r(R(\Phi)) \subset \Phi_{0}$. Observe $R(\Phi) \subset \Phi_{0} \cap \Psi_{0}$, and that for $\alpha \in \Psi_{0} \sim R(\Phi), \gamma_{\alpha} \in M_{0}$. For $I \subset R(\Phi)$, set $\gamma_{I}=\prod_{a \in I} \gamma_{\alpha}$.

LemMa 3.4. For $I \subset R(\Phi), \gamma_{I} \notin M_{0}$.

Proof. Suppose $\gamma_{I} \in M_{0}$. As $\gamma_{I}$ is in the center of $M, \gamma_{I}$ is in the center of $M_{0}$. Thus there is a $Z \in t$ such that $\gamma_{I}=\exp Z$. Hence

$$
Z=\sum_{\beta \in \Phi_{(x)}} i c_{\beta} H_{\beta} .
$$

As $\gamma_{I} \exp -Z=e($ see $[\mathbf{L}$, p. 77]),

$$
2 \pi i \sum_{\lambda \in I} H_{\lambda} /\langle\lambda, \lambda\rangle-\sum_{\beta \in \Phi_{(x)}} c_{\beta} i H_{\beta}=4 \pi i \sum_{\alpha \in \Phi} n_{\alpha} H_{\alpha} /\langle\alpha, \alpha\rangle
$$

for integers $n_{\alpha}$. However as the $H_{\alpha}$ 's are linearly independent we have a contradiction.

Let $\bar{\Gamma}(\Phi)=\left\langle\gamma_{\alpha}: \alpha \in R(\Phi)\right\rangle$. Since $\bar{\Gamma}(\Phi)$ is contained in the center of $M, 3.1$ through 3.4 yield

TheOREM 3.5. $M=\bar{\Gamma}(\Phi) \cdot M_{0}=\bar{\Gamma}(\Phi) \times M_{0}$, and $M / M_{0}=\bar{\Gamma}(\Phi)$. Furthermore, $\bar{\Gamma}(\Phi)$ is the direct product of $|R(\Phi)|$-copies of $\mathbf{Z}_{2}$.

In terms of the Satake diagram, $\alpha \in R(\Phi)$, if $\alpha$ is not adjacent to a black dot and for no $\beta \in \Phi$ is $\alpha$ joined to $\beta$ by a double headed arrow.

Corollary 1. Suppose $I \subset \Phi_{0}$. Then $P(I) / P_{0}(I)=\bar{\Gamma}(\Phi, I)$, where $P_{0}(I)$ is the connected component of the identity of $P(I)$, and

$$
\bar{\Gamma}(\Phi, I)=\left\langle\gamma_{\alpha}: \alpha \in I \cap R(\Phi)\right\rangle .
$$


Corollary 2. Suppose $\pi: G \rightarrow G L(V)$ is a finite dimensional irreducible representation with highest weight $\Lambda$ and nonzero highest weight vector $v \in V^{N}$. Then $v$ is an $M$-fixed vector if and only if the following conditions hold:

(a) $\langle\Lambda, \alpha\rangle=0$ if $\alpha \in \Phi_{00}$;

(b) $\langle\Lambda, \alpha\rangle=\langle\Lambda, \beta\rangle$ if $\alpha, \beta \in \Phi$ are joined by a double headed arrow in the Satake diagram; and

(c) $\langle\Lambda, \alpha\rangle /\langle\alpha, \alpha\rangle$ is a nonnegative integer for $\alpha \in R(\Phi)$.

This corollary is merely a restatement of Helgason's condition that:

(a) $\pi(x) v=0$ for all $X \in \mathfrak{m}$; and,

(b) $\langle\Lambda, \lambda\rangle /(\lambda, \lambda)$ is a nonnegative integer for $\lambda \in \Phi_{0}$.

From the Satake diagrams of real forms of complex groups we obtain

Corollary 3. i. $R(\Phi)$ is empty if $G$ is complex, $S U^{*}(2 n), S U(p, q)(p<q)$, $\operatorname{Spin}_{0}(1, n), S O^{*}(4 n+2)(n \geqslant 1), S p(p, q), E_{6(-14)}, E_{6(-26)}, F_{4(-20)}$.

ii. $R(\Phi)=\Phi$ if $G$ is split.

iii. For the remaining groups the following chart describes $G$ and $|R(\Phi)|$.

\begin{tabular}{|c|c|c|c|c|c|c|}
\hline$G$ & $S U(p, p)$ & $\operatorname{Spin}(p, q)(p<q)$ & $E_{6(2)}$ & $E_{7(-5)}$ & $E_{7(-25)}$ & $E_{8(-24)}$ \\
\hline$|R(\Phi)|$ & 1 & $p-1$ & 2 & 2 & 1 & 2 \\
\hline
\end{tabular}

REMARK. If $G / K$ is a Hermitian symmetric space not of tube type, Schlichtkrull proved that $M=M_{0}$ in [S].

4. Some Lie algebra cohomology. Suppose $U$ is closed subgroup of $K$, and $P$ is a parabolic subgroup containing $B$ such that:

1. $\mathfrak{u}+\mathfrak{p}=\mathfrak{g}$; and

2. $\mathfrak{u} \cap \mathfrak{p}$ is an ideal in the reductive part of $\mathfrak{p}$.

From these assumptions we have that there is an ideal $\mathfrak{l} \subset \mathfrak{f} \cap p$ such that:

3. $\mathfrak{u} \cap \mathfrak{p} \oplus \mathfrak{l}=\mathfrak{f} \cap \mathfrak{p}$ as Lie algebras, and

4. $\mathfrak{u}+\mathfrak{l}=\mathfrak{f}, \mathfrak{u} \cap \mathfrak{l}=(0)$.

To continue we recall some facts concerning Lie algebra cohomology. For $\mathfrak{m}$ a compact Lie algebra, let $H^{k}(\mathrm{~m})$ be the $k$ th cohomology group with real coefficients and set $d_{k}=\operatorname{dim} H^{k}(\mathfrak{w})$. For $\mathfrak{t}$ real, the Poincaré polynomial $P(\mathfrak{w}, t)$ is given by the formula

$$
P(\mathfrak{w}, t)=\sum_{t \geqslant 0} d_{k} t^{k} .
$$

It is well known that if $w$ is of rank $r$, there are $r$ integers $n_{1} \leqslant n_{2} \leqslant \cdots \leqslant n_{r}$ :

$$
P(\mathfrak{w}, t)=\prod_{k=1}^{r}\left(1+t^{2 n_{i}+1}\right)
$$

with

$$
\begin{aligned}
& \#\left\{i: n_{i}=0\right\}=\text { dimension of the center of } \mathfrak{w}, \\
& \#\left\{i: n_{i}=1\right\}=\text { number of simple ideals of } \mathfrak{w} .
\end{aligned}
$$


If $\mathfrak{u}_{1}$ and $\mathfrak{u}_{2}$ are subalgebras of $\mathfrak{w}$ and $\mathfrak{w}=\mathfrak{u}_{1}+\mathfrak{u}_{2}$, from [O], we have

$$
P(\mathfrak{w}, t) P\left(\mathfrak{u}_{1} \cap \mathfrak{u}_{2}, t\right)=P\left(\mathfrak{u}_{1}, t\right) P\left(\mathfrak{u}_{2}, t\right)
$$

where $P((0), t) \equiv 1$. Hence if $\mathfrak{k}, \mathfrak{u}$ and $\mathfrak{l}$ are as above,

$$
P(\mathfrak{f}, t)=P(\mathfrak{u}, t) P(\mathfrak{l}, t) \text {. }
$$

From our above equations we have the following.

Proposition 4.1. If $K$ is simple or abelian and $U$ and $P$ are as above $U=K$ and $P=B$.

Lemma 4.2. Suppose $\mathfrak{f}_{1}$ and $\mathfrak{f}_{2}$ are two nontrivial minimal ideals in $\mathfrak{f}$ and $\mathfrak{f}=\mathfrak{f}_{1} \oplus \mathfrak{f}_{2}$. Let $\mathfrak{u}, \mathfrak{p}, \mathfrak{l}, \mathfrak{f}$ and $\mathfrak{g}$ satisfy $1,2,3$, and 4 above with $\mathfrak{u} \neq \mathfrak{f}$.

If $\operatorname{dim} \mathfrak{f}_{1} \geqslant \operatorname{dim} \mathfrak{f}_{2}, \mathfrak{u} \cong \mathfrak{f}_{1}$ and $\mathfrak{l} \cong \mathfrak{f}_{2}$. Moreover, if $\operatorname{dim} k_{1}>\operatorname{dim} \mathfrak{f}_{2}, \mathfrak{u}=\mathfrak{f}_{1}$.

Proof. Let $Q_{i}: \mathfrak{f} \rightarrow \mathfrak{f}_{i}$ be the projection which is 0 on $\mathfrak{f}_{j}$ if $j \neq i$.

Assume first that $\operatorname{dim} \mathfrak{l}>\operatorname{dim} \mathfrak{u}$. From equations (4.1) to (4.5) and our assumptions on $\mathfrak{f}, \mathfrak{I}$ and $\mathfrak{u}$ contain no proper ideals. Thus $Q_{i}$ restricted to $\mathfrak{l}$ or $\mathfrak{u}$ is either 0 or an injection. As $2 \operatorname{dim} \mathfrak{l}>\operatorname{dim} \mathfrak{f}, \operatorname{dim} \mathfrak{l}>\operatorname{dim} \mathfrak{f}_{2}$, and hence $Q_{2}(\mathfrak{l})=(0)$. Thus $\mathfrak{l} \subset \mathfrak{f}_{1}$. It follows that $\mathfrak{u} \cong \mathfrak{f}_{2}$ and $\mathfrak{l}=\mathfrak{f}_{1}$. As $\mathfrak{l}$ is an ideal in $\mathfrak{f}, \mathfrak{g}^{\mathfrak{l}}=\mathfrak{f}^{\mathfrak{l}}$, and hence $\mathfrak{l}$ does not centralize any nonzero element of $a$. This contradicts our assumption that $\operatorname{dim} l>\operatorname{dim} u$.

As $\operatorname{dim} \mathfrak{l} \leqslant \operatorname{dim} u$ it follows that $Q_{2}(\mathfrak{u})=(0)$, if $\operatorname{dim} \mathfrak{f}_{1}>\operatorname{dim} \mathfrak{f}_{2}$ and hence $\mathfrak{l} \cong \mathfrak{f}_{2}$ and thus $\mathfrak{u}=\mathfrak{f}_{1}$. Now by classification, $\operatorname{dim} \mathfrak{f}_{1}=\operatorname{dim} \mathfrak{f}_{2}$ if and only if $\mathfrak{f}_{1} \cong \mathfrak{f}_{2}$. In this case, by taking projections, we see that $\mathfrak{u} \cong \mathfrak{l} \cong \mathfrak{f}_{i}$.

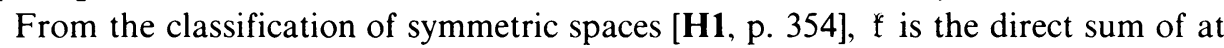
most 4 ideals. The only case where $f$ is the direct sum of 4 ideals occurs when $G=\operatorname{Spin}_{0}(4,4)$. The only cases where $f$ is the direct sum of 3 minimal ideals occurs when $G=S U(p, q)(2 \leqslant p \leqslant q)$, or when $G=\operatorname{Spin}_{0}(4, q)(q \neq 1,4)$.

Lemma 4.3. Let $\mathfrak{u}, \mathfrak{p}, \mathfrak{g}, \mathfrak{f}$ and $\mathfrak{l}$ satisfy conditions $1,2,3$, and 4 . If $\mathfrak{u}$ is an ideal in $\mathfrak{f}$ anc $\mathfrak{f}_{1}$ is a minimal ideal in $\mathfrak{f}^{\mathfrak{t}}$ such that $2 \operatorname{dim} \mathfrak{f}_{1}>\operatorname{dim} \mathfrak{f}, \mathfrak{f}_{1} \subset \mathfrak{u}$.

Proof. Let $\mathfrak{f}=\mathfrak{f}_{1} \oplus \mathfrak{f}^{\prime}$ and suppose $Q_{1}: \mathfrak{f} \rightarrow \mathfrak{f}_{1}$ is the projection which is 0 on $\mathfrak{f}^{\prime}$.

Assume $\mathfrak{f}_{1} \not \subset \mathfrak{u}$. Hence $Q_{1}(\mathfrak{l})=\mathfrak{f}_{1}$, and so $\mathfrak{I}$ contains an ideal $\mathfrak{l}_{1}$ isomorphic to $\mathfrak{f}_{1}$. As $Q^{\prime}\left(\mathfrak{l}_{1}\right)=0$ we have that $\mathfrak{l}_{1}=\mathfrak{f}_{1}$. Hence $\mathfrak{f}_{1} \subset \mathfrak{l}$ and as $\mathfrak{g}^{\mathfrak{f}_{1}}=\mathfrak{f}^{\mathfrak{f}_{1}}$ we have that $\mathfrak{l}$ centralizes no element of $\mathfrak{a}$. This contradicts the definition of $\mathfrak{p}$.

Corollary. Suppose $\mathfrak{g}, \mathfrak{f}, \mathfrak{u}, \mathfrak{p}$ and $\mathfrak{l}$ satisfy the hypotheses of Lemma 4.3. If $G=S U(p, q)$ with $p<q, S U(q) \subset U$. If $G=\operatorname{Spin}_{0}(p, q)$ with $p<q \neq 4, \operatorname{Spin}(q)$ $\subset U$.

In the next three sections we shall classify all reciprocity pairs in $G$. To accomplish this, the following notation will prove useful. For $\alpha \in \Sigma_{0}^{+}$, set

$$
\mathfrak{f}_{\alpha}=\mathfrak{f} \cap\left(\mathfrak{g}_{\alpha}+\mathfrak{g}_{-\alpha}\right)=\left\{x+\theta x: x \in \mathfrak{g}_{\alpha}\right\} .
$$

If $I \subset \Phi_{0}$ let $\mathfrak{f}(I)$ be the Lie algebra generated by $\left\{\mathfrak{f}_{\alpha}: \alpha \in \Phi_{0} \sim I\right\}$, and let $\mathfrak{f}_{M}(I)$ be the Lie algebra generated by $\mathfrak{f}(I)$ and $m$. Observe that $\mathfrak{f}_{M}(I)=\mathfrak{f} \cap \mathrm{m}(I)$. Let $\mathfrak{g}(I)$ be the Lie algebra generated by $\left\{\mathfrak{g}_{ \pm \alpha}: \alpha \in \Phi_{0} \sim I\right\}$, and $\mathfrak{g}_{M}(I)$ the Lie 
algebra generated by $\mathfrak{g}(I)$ and $\mathfrak{m}$. Then $\mathfrak{m}(I)=\mathfrak{g}_{M}(I)$. From Corollary 1 of Theorem 3.5, we have the following:

Proposition 4.4. If $J=R(\Phi) \sim I, \Gamma(\Phi, J) \subset K(I)$ where $K(I)$ is the analytic group with lie algebra $k(I)$.

We conclude this section with the following:

Lemma 4.5. Suppose $(U, P)$ is a reciprocity pair in $G$, and $Q^{\prime}, Q^{\prime \prime} \in \mathbf{P}$ with $Q^{\prime} \subsetneq P \subsetneq Q^{\prime \prime}$. Then neither $\left(U, Q^{\prime}\right)$ nor $\left(U, Q^{\prime \prime}\right)$ is a reciprocity pair.

Proof. Now $\mathfrak{f}^{\prime} \cap q^{\prime} \subsetneq \mathfrak{f} \cap \mathfrak{p} \subsetneq \mathfrak{f} \cap q^{\prime}$.

Assume $\left(U, Q^{\prime}\right)$ is a reciprocity pair. Then

$$
\mathfrak{f} \cap \mathfrak{q}^{\prime}=\left(\mathfrak{u} \cap \mathfrak{q}^{\prime}\right) \oplus \mathfrak{l}^{\prime}=(\mathfrak{u} \cap \mathfrak{m}) \oplus \mathfrak{l}^{\prime} \subsetneq \mathfrak{u} \cap \mathfrak{m} \oplus \mathfrak{l} .
$$

Hence $\operatorname{dim} \mathfrak{l}^{\prime}<\operatorname{dim} \mathfrak{l}$, and $\operatorname{so} \mathfrak{f} \neq \mathfrak{u}+\mathfrak{l}^{\prime}$, which contradicts our assumption.

Assume now that $\left(U, Q^{\prime \prime}\right)$ is a reciprocity pair. Then

$$
\mathfrak{f} \cap \mathfrak{p}=\mathfrak{u} \cap \mathfrak{m} \oplus \mathfrak{l} \subsetneq \mathfrak{u} \cap \mathfrak{m} \oplus \mathfrak{l}^{\prime \prime}=\mathfrak{l} \cap \mathfrak{q}^{\prime \prime} .
$$

Hence $\operatorname{dim} \mathfrak{l}<\operatorname{dim} \mathfrak{l}^{\prime \prime}$ and we must have $\mathfrak{u} \cap \mathfrak{l}^{\prime \prime} \neq(0)$, which contradicts our assumption.

5. Classification of exceptional types. Throughout this section, we shall assume $G$ is a simple group of exceptional type and $K=K_{1} \cdot K_{2}$ where $K_{1}$ and $K_{2}$ are simple analytic groups. (The notation $K=K_{1} \cdot K_{2}$ means that $K_{1} \cap K_{2}$ is finite and $\mathfrak{f}=\mathfrak{f}_{1} \oplus \mathfrak{f}_{2}$. We call $K$ the local direct product of $K_{1}$ and $K_{2}$.) From [H2, p. 354], $G$ is one of five groups, and if we assume that $\operatorname{dim} K_{1} \geqslant \operatorname{dim} K_{2}, K_{2}=S U(2)$ in every case. In all cases $K_{1} \cap K_{2}=\mathbf{Z}_{2}$.

(A) If $G$ is of type $G_{2}$ and $P$ is a parabolic subgroup, $\operatorname{dim} G / P \geqslant 5$. As $K_{1}=S U(2)$ the only reciprocity pair in $G$ is $(K, B)$.

(B) For the remaining four groups $G$ has restricted root diagram of type $F_{4}$ and $\operatorname{dim} K_{1}>\operatorname{dim} K_{2}$. Hence if $(U, P)$ is a reciprocity pair different from $(K, B)$, $U=K_{1}$. The following chart gives the pairs $\left(G, K_{1}\right)$ :

\begin{tabular}{|c|c|c|c|c|}
\hline$G$ & $F_{4(4)}$ & $E_{6(2)}$ & $E_{7(-5)}$ & $E_{8(-24)}$ \\
\hline$K_{1}$ & $\operatorname{Sp}(3)$ & $S U(6)$ & $\operatorname{Spin}(12)$ & $E_{7}$ \\
\hline
\end{tabular}

If $\Phi_{0}=\left\{\mu_{i}: 1 \leqslant i \leqslant 4\right\}$ let

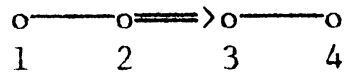

denote the restricted root diagram. It will be useful to list the Satake diagrams of the different $G$. We put $i$ next to the double headed arrow joining $\alpha$ and $\beta$ if $r(\alpha)=r(\beta)=\mu_{i}$. If $\alpha \in \Phi$ is the only simple root for which $r(\alpha)=\mu_{i}$, we put an $i$ under the corresponding dot.

1. $G$ of type $F_{4}$ :

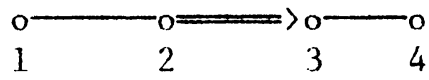


2. $G$ of type $E_{6}$ :

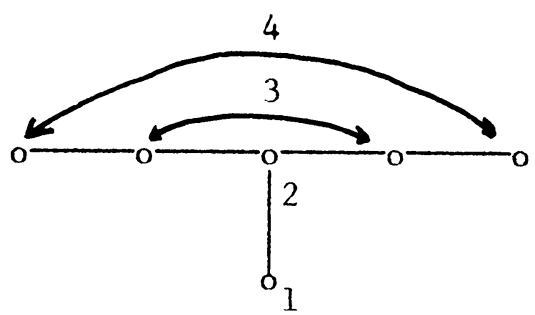

3. $G$ of type $E_{7}$ :

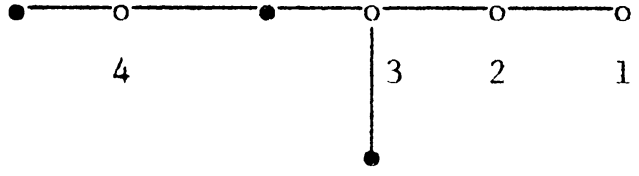

4. $G$ of type $E_{8}$ :

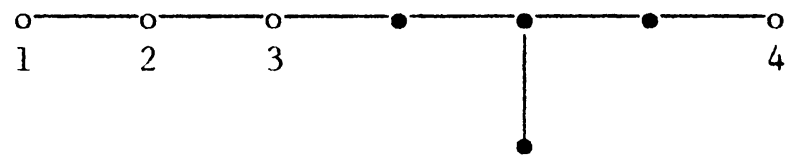

LEMMA 5.1. If $G$ has restricted root diagram of type $F_{4}, \mathfrak{f}_{1}+\mathfrak{p}(I)=\mathfrak{q}$, if and only if $I \subset\{3,4\}$.

Proof. It suffices to prove that $\mathfrak{f}_{M}(I)+\mathfrak{f}_{1}=\mathfrak{f}$, if and only if $I \subset\{3,4\}$. We shall actually show $\mathfrak{f}=\mathfrak{f}_{1}+\mathfrak{f}\{3,4\}$.

i. If $G$ is of type $F_{4}, \mathfrak{m}=(0)$ and $\mathfrak{f}_{M}(I)=\mathfrak{f}(I)$. Now $\mathfrak{k}\{1\}=\mathfrak{u}(3)$, and so

$$
\mathfrak{s} \mathfrak{u}(2)=\mathfrak{f}\{1,2\} \subset[\mathfrak{f}\{1\}, \mathfrak{f}\{1\}]=\mathfrak{S} \mathfrak{u}(3) \subset \mathfrak{K}_{1} .
$$

Now $\mathfrak{f}\{2\}=\mathfrak{f}\{1,2\} \oplus \mathbf{R}$. Hence, if $I=\{1\}$ or $\{2\}, \mathfrak{f}_{1}+\mathfrak{f}(I) \neq \mathfrak{f}$. As $\mathfrak{f}(\phi)=\mathfrak{f}$ and $\mathfrak{f}\{1,2\} \subset \mathfrak{f}_{1}, \mathfrak{f}\{3,4\}=\mathfrak{s} \mathfrak{u}(2) \not \subset \mathfrak{f}_{1}$. Thus $\mathfrak{f}_{1}+\mathfrak{f}\{3,4\}=\mathfrak{f}$.

ii. If $G$ is of type $E_{6}, \mathfrak{f}_{M}\{1,2\}=\mathfrak{s} \mathfrak{u}(3) \subset \mathfrak{f}_{1}$ and again $\mathfrak{f}\{3,4\}=\mathfrak{s u}(2) \not \subset \mathfrak{f}_{1}$. Thus $\mathfrak{f}_{1}+\mathfrak{f}\{3,4\}=\mathfrak{f}$. As $\mathfrak{f}_{M}\{1\}=\mathfrak{s}(\mathfrak{u}(3) \oplus \mathfrak{u}(3))$, $\mathfrak{f}\{1\}+\mathfrak{f}_{1} \neq \mathfrak{f}$. As $\mathfrak{f}_{M}\{2\}=\mathbf{R}$ $\oplus \mathfrak{f}\{1,2\}, \mathfrak{f}\{2\}+\mathfrak{f}_{1} \neq \mathfrak{f}$.

iii. If $G$ is of type $E_{7}, \mathfrak{f}_{M}\{1,2\}=\mathfrak{s p}(3) \subset \mathfrak{f}_{1}$, and so, as $\mathfrak{f}\{3,4\}=\mathfrak{s} \mathfrak{u}(2) \not \subset \mathfrak{f}_{1}$, $\mathfrak{f}\{3,4\}+\mathfrak{f}_{1}=\mathfrak{f}$. As $\mathfrak{f}_{M}\{1\}=\mathfrak{u}(6), \mathfrak{f}_{M}\{1\}+\mathfrak{f}_{1} \neq \mathfrak{f}$. As $\mathfrak{f}_{M}\{2\}=\mathbf{R} \oplus \mathfrak{s} \mathfrak{p}(3)$, $\mathfrak{f}_{M}\{2\}+\mathfrak{f}_{1} \neq \mathfrak{f}$.

iv. If $G$ is of type $E_{8}, \mathfrak{f}_{M}\{1,2\}=\mathfrak{f}_{4} \subset \mathfrak{f}_{1}$, and hence $\mathfrak{f}\{3,4\}=\mathfrak{s} \mathfrak{u}(2) \not \subset \mathfrak{f}_{1}$. Thus $\mathfrak{f}_{1}+\mathfrak{f}\{3,4\}=\mathfrak{f}$. As $\mathfrak{f}_{M}\{2\}=\mathfrak{f}_{4} \oplus \mathbf{R}, \mathfrak{f}_{M}\{2\}+\mathfrak{f}_{1} \neq \mathfrak{f}$. As $\mathfrak{f}_{M}\{1\}=\mathfrak{e}_{6} \oplus \mathbf{R}$, $\mathfrak{f}_{M}\{1\}+\mathfrak{f}_{1} \neq \mathfrak{f}$. This completes the proof of the lemma.

For $i \leqslant 4$, set $\gamma_{i}=\gamma_{\lambda_{i}}$. Then $\bar{\Gamma}=\left\langle\gamma_{i}: i \leqslant 4\right\rangle$. Consider the parabolic group $P\{3,4\}$. From Corollary 1 of Theorem 3.5, $P\{3,4\}=\left\langle\gamma_{3}, \gamma_{4}\right\rangle \times P_{0}\{3,4\}$ if $G$ is of type $F_{4}$, and $P\{3,4\}$ is connected in the remaining cases. In particular, $\left\langle\gamma_{3}, \gamma_{4}\right\rangle \subset$ 
$M_{0} \subset K_{1}$ if $G$ is of type $E_{6}, E_{7}$, or $E_{8}$ while if $G$ is of type $F_{4}, M_{0}=\{e\}$ and $\left\langle\gamma_{3}, \gamma_{4}\right\rangle \subset K_{1}$. If $G$ is of type $E$,

$$
M\{3,4\}=\operatorname{SL}(3, \mathbf{R}) \times M_{0} .
$$

If $G$ is of type $F_{4}$,

$$
M\{3,4\}=\operatorname{SL}(3, \mathbf{R}) \times\left\langle\gamma_{3}, \gamma_{4}\right\rangle .
$$

Let $T: K \rightarrow K_{2} / \mathbf{Z}_{2}$, be the projection with kernel $K_{1}$. As $T: M\{3,4\} \cap K \rightarrow$ $K_{2} / \mathbf{Z}_{2}$, is a surjective homomorphism, $T$ is an isomorphism on the $S O(3)$ factor of $K \cap M\{3,4\}$. Thus in any case $\left(K_{1}, P\{3,4\}\right)$ is a reciprocity pair in $G$.

THEOREM 5.2. The only reciprocity pairs in $G$ are $(K, B)$ and $\left(K_{1}, P\{3,4\}\right)$.

Remark. In every case, $M\{3,4\}=\operatorname{SL}(3, \mathbf{R}) \times M_{1}$, where $M_{1}=K_{1} \cap M$.

6. Hermitian symmetric spaces. When $G / K$ is a Hermitian symmetric space, $K$ has a one dimensional center. Then $K_{1}=[K, K]$, the commutator subgroup of $K$, is an analytic group with Lie algebra $[\mathfrak{k}, \mathfrak{f}]$. In this section, we determine all reciprocity pairs $\left(K_{1}, P\right)$. After first recalling some of the fundamental structure of Hermitian symmetric spaces, we list, giving only references, results which are important to our considerations. The determination of the reciprocity pairs relies upon distinguishing between the tube type and the nontube type case.

If $z$ is the center of $\mathfrak{f}$, there is a $Z \in \mathfrak{z}$ such that $(\operatorname{ad} Z)^{2}=-I$, on $\mathfrak{p}$. Fixing $\sqrt{-1}$, let $\mathfrak{p}_{ \pm}$be the $\pm \sqrt{-1}$-eigenspace of ad $Z$ on $\mathfrak{p}_{\mathbf{C}}$. Then

$$
\mathfrak{g}_{\mathbf{C}}=k_{\mathbf{C}}+\mathfrak{p}_{+}+\mathfrak{p}_{-}, \quad\left[\mathfrak{p}_{ \pm}, \mathfrak{p}_{ \pm}\right]=0, \quad\left[\mathfrak{p}_{+}, \mathfrak{p}_{-}\right]=\mathfrak{f}_{\mathbf{C}}, \quad\left[\mathfrak{f}_{\mathbf{C}}, \mathfrak{p}_{ \pm}\right]=\mathfrak{p}_{ \pm} .
$$

Now $\mathfrak{f}^{*}$ contains a Cartan subalgebra $\mathfrak{h}^{0}$ of $\mathfrak{g}$, and if $\Delta$ is the set of roots of $\mathfrak{g}_{\mathbf{C}}$ with respect to $\mathfrak{h}_{\mathbf{C}}^{0}, \Delta=\Delta_{c} \cup \Delta_{n}$ where $\Delta_{c}$ is the set of compact roots and $\Delta_{n}$ is the set of noncompact roots. Let $\Delta_{n}^{+} \subset \Delta_{n}$ be the set of roots $\alpha$ for which $\alpha(Z)=\sqrt{-1}$. Then $\Delta_{n}=\Delta_{n}^{+} \cup\left(-\Delta_{n}^{+}\right)$and there is an ordering $\Delta_{+}$of $\Delta$ so that $\Delta_{n}^{+}=\Delta_{n} \cap \Delta^{+}$. As in [K-W] select $\left\{\nabla_{1}, \ldots, \nabla_{l}\right\} \subset \Delta_{n}^{+}$strongly orthogonal roots. Let $H_{i}^{0} \in \sqrt{-1} \mathfrak{h}^{0}$ be the element for which $B\left(H_{i}^{0}, H\right)=\nabla_{i}(H)$ for all $H \in \mathfrak{h}_{\mathbf{C}}^{0}$. Set

$$
\mathfrak{h}^{-}=\sum_{i=1}^{l} \mathbf{R} H_{i}^{0}
$$

and let $\mathfrak{h}^{+}$be its orthogonal complement in $\sqrt{-1} \mathfrak{h}$.

For $W \in \mathfrak{g}_{\mathbf{C}}$, let $\bar{W}$ be its congugate with respect to $\mathfrak{g}$. Then $\overline{\mathfrak{g}}_{\alpha}=\mathfrak{g}_{-\alpha}$, for all $\alpha \in \Delta$, and for $i \leqslant l$, pick $E_{i} \in \mathfrak{g}_{\nabla}$, so that

$$
2 H_{i}=\left[E_{i}, \bar{E}_{i}\right]=2 H_{i}^{0} /\left\langle\nabla_{i}, \nabla_{i}\right\rangle .
$$

Set $X_{i}^{0}=E_{i}+\bar{E}_{i}$ and $Y^{0}=\sqrt{-1}\left(E_{i}-\bar{E}_{i}\right)$. Then

$$
\mathfrak{g}_{i}=\mathbf{R} \sqrt{-1} H_{i}^{0}+\mathbf{R} X_{i}^{0}+\mathbf{R} Y_{i}^{0}
$$

is a three dimensional simple subalgebra of $\mathfrak{g}$ isomorphic to $\mathfrak{s} \mathfrak{l}(2, \mathbf{R})$, and $\mathfrak{a}=$ $\sum_{j=1}^{\prime} \mathbf{R} Y_{j}$, is a maximal abelian subalgebra of $\mathfrak{p}$. If

$$
c=\exp \left(\pi / 4 \sqrt{-1} \sum_{i=1}^{l} X_{j}^{0}\right)
$$


$c$ is the generalized Cayley transforms of Korányi-Wolf, and

$$
\text { Ad } c: X_{i}^{0} \rightarrow X_{i}^{0}, \quad 2 H_{i} \rightarrow Y_{i}^{0}, \quad Y_{i}^{0} \rightarrow-2 H_{i} .
$$

Let $j:\left(\sqrt{-1} \mathfrak{h}^{0}\right)^{*} \rightarrow\left(\mathfrak{h}^{-}\right)^{*}$, be the restriction map, and $\tau:\left(\mathfrak{h}^{-}\right)^{*} \rightarrow a^{*}$ the dual of the map Ad $c: a \rightarrow \mathfrak{h}^{-}$. Then, set $\Delta_{0}=j(\Delta) \sim\{0\}$ and $\Delta_{0}^{+}=j\left(\Delta^{+}\right) \sim\{0\}$. After possibly renumbering $\left\{\nabla_{1}, \ldots, \nabla_{l}\right\}$ we recall the following result.

1. (Moore, $[\mathbf{M}])$ The set of elements of $\Delta_{0}^{+}$is one of the following sets:

Case I. $\left\{\nabla_{i}, \frac{1}{2}\left(\nabla_{j} \pm \nabla_{k}\right): 1 \leqslant i \leqslant l, 1 \leqslant j<k \leqslant l\right\}$.

Case II: $\left\{\frac{1}{2} \nabla_{i}, \nabla_{i}, \frac{1}{2}\left(\nabla_{j} \pm \nabla_{k}\right): 1 \leqslant i \leqslant l, 1 \leqslant j<k \leqslant l\right\}$.

Furthermore, if $\nabla \in \Delta_{c}^{+}, j(\nabla)$ is either $\frac{1}{2} \nabla_{i}$ or $\frac{1}{2}\left(\nabla_{j}-\nabla_{k}\right)$. If $\nabla \in \Delta_{n}^{+}, j(\nabla)$ is either $\frac{1}{2} \nabla_{i}, \nabla_{i}$, or $\frac{1}{2}\left(\nabla_{j}+\nabla_{k}\right)$.

The roots $\nabla_{1}, \ldots, \nabla_{1}$ belong to the set of longest roots of $\Delta$. In case II, only one root length occurs in $\Delta$.

Unless $\mathfrak{h}^{+}=0, \nabla_{1}, \ldots, \nabla_{1}$ are the only restricted roots of multiplicity one.

The space $G / K$ is of tube type if and only if $\Delta_{0}$ belongs to Case I.

Set $\alpha_{i}=\tau\left(\nabla_{i}\right)$ for $i \leqslant l$. From $\S 3$,

$$
\Psi_{0}=\left\{\frac{1}{2}\left(\alpha_{i}+\alpha_{i+1}\right): 1 \leqslant i<l\right\} \cup\left\{\alpha_{l}\right\} .
$$

If $\beta_{i}=\frac{1}{2}\left(\alpha_{i}+\alpha_{i+1}\right)$, and $\gamma_{i}=\exp 2 \pi \sqrt{-1} Y_{i}$, we have

$$
\exp \left(2 \pi \sqrt{-1} H_{\beta_{t}}\right)=\gamma_{i} \gamma_{i+1} \text {. }
$$

Thus we obtain

2. The group $\bar{\Gamma}=\left\langle\gamma_{i}: i \leqslant l\right\rangle$.

3. $[\mathbf{J}] \mathfrak{g}^{M}=\mathfrak{g}_{1} \oplus \cdots \oplus \mathfrak{g}_{l} \oplus \mathrm{m}$.

Set $Z^{+}=\sqrt{-1} \sum_{j=1}^{\prime} H_{j}$.

4. [K-W] i. There is a $Z_{0} \in \mathfrak{m}$, such that $Z=Z^{+}+Z_{0}$.

ii. The space $G / K$ is tube type, if and only if $Z_{0}=0$.

Hence $m \subset \mathfrak{f}_{1}$, if and only if $G / K$ is tube type.

From this and the lemma we now obtain

Proposition 6.1. Suppose $G / K$ is not tube type. Then $\left(K_{1}, P\right)$ is a reciprocity pair, if and only if $P=B$.

5. [S] Suppose $G / K$ is not tube type. Then $M$ is connected and $K_{1} \cap M$ is connected. The connectedness of $M$ also follows from Corollary 3.i of $\S 3$.

Let $\bar{\Gamma}_{e}=\left\langle\gamma_{i} \gamma_{i+1}: i \leqslant l-1\right\rangle$.

6. [S] For any $G, K_{1} \cap \bar{\Gamma}=\bar{\Gamma}_{e}$.

Suppose now that $G / K$ is tube type. As $\mathfrak{m} \subset \mathfrak{f}_{1}$, if $M_{1}=K_{1} \cap M$, we have $\left[M: M_{1}\right]=\left[\bar{\Gamma}_{i} \bar{\Gamma}_{e}\right]=2$. Hence $\left(K_{1}, B\right)$ is not a reciprocity pair. Now

$$
\Phi_{0}=\left\{\frac{1}{2}\left(\alpha_{i}+\alpha_{i+1}\right): i \leqslant l-1\right\} \cup\left\{\alpha_{l}\right\} \text {. }
$$

Let $S_{0}=\left\{\frac{1}{2}\left(\alpha_{i}+\alpha_{i+1}\right): i \leqslant l-1\right\}$.

Proposition 6.2. If $G$ is of tube type $\mathbf{P}\left(K_{1}\right)=\left\{P\left(S_{0}\right)\right\}$.

Proof. Let $G_{i}$ be the analytic group with Lie algebra $g_{i}$. From $[\mathbf{S}], G_{i} \cong S L(2, \mathbf{R})$ with $\gamma_{i}$ mapping to $-I$ by this isomorphism, and $K_{1} \cap G_{i}=\{e\}$. Then the reductive part of $P\left(S_{0}\right)$ is $M \cdot G_{l}=M_{1} \cdot G_{l}$. As $\left[\mathrm{m}, \mathfrak{g}_{l}\right]=0$, and $\gamma_{i} g=g \gamma_{i}$ for any $g \in G_{l}$, 
$M_{1}=K_{1} \cap P\left(S_{0}\right)$, is normal in $M G_{l}$. Actually, $M \cdot G_{l}=M_{1} \times G_{l}$. Hence $P\left(S_{0}\right) \in$ $\mathbf{P}\left(K_{1}\right)$.

To show that $P(S) \notin \mathbf{P}\left(K_{1}\right)$, for $S_{0} \neq S \subset \Phi_{0}$, it suffices to show that $G \neq$ $K_{1} P\left(\left\{\alpha_{l}\right\}\right)$. However, $G / P\left\{\alpha_{l}\right\}$ is the Shilov boundary of $G / K$ and from [K-W] we have that

$$
K_{1} / K_{1} \cap P\left(\left\{\alpha_{l}\right\}\right) \neq K / K \cap P\left(\left\{\alpha_{l}\right\}\right) .
$$

REMARK. If $G / K$ is not of tube type, it was shown in [S] that for $S^{\prime}=\left\{\exp t Z_{0}\right.$ : $t \in \mathbf{R}\}, K_{1} \cap S^{\prime}=\{e\}$, and hence $M=M_{1} \cdot S^{\prime}=M_{1} \times S^{\prime}$.

7. The Lorentz groups In this section we examine the following three cases:

i. $G=\operatorname{Sp}(p, q)(2 \leqslant p \leqslant q)$, and $K_{1}=\operatorname{Sp}(q)$;

ii. $G=\mathrm{SU}(p, q)(2 \leqslant p \leqslant q)$, and $K_{1}=\mathrm{SU}(q)$ or $U(q)$; and

iii. $G=\operatorname{Spin}_{0}(p, q)(3 \leqslant p \leqslant q)$, and $K_{1}=\operatorname{Spin}(q)$.

Rather than iii, it will be more convenient to consider iii'.

$$
G=S O_{0}(p, q)(3 \leqslant p \leqslant q), \text { and } K_{1}=S O(q) .
$$

It is clear that the problem of finding all parabolic groups $P$ for which $K \cdot P=G$ and $K_{1} \cap P$ is normal in the reductive part of $P$ are equivalent for iii and iii'.

In cases $\mathrm{i}$, ii, and iii', $G$ is the group of all matrices

$$
X=\left(\begin{array}{ll}
A & B \\
C & D
\end{array}\right)
$$

where $A$ is a $p \times p$ matrix, $B$ is a $p \times q$ matrix, $C$ is a $q \times p$ matrix and $D$ is a $q \times q$ matrix with entries in the quatermions, complex numbers, or reals according as $G$ is in case $\mathrm{i}$, ii, or iii' where

a.

$$
X^{-1}=\left(\begin{array}{cc}
A^{*} & -C^{*} \\
-B^{*} & D^{*}
\end{array}\right) ;
$$

b. $\operatorname{det} X=1$ in case ii; and,

c. $G$ is the connected component of the identity of the group given by a, if $G$ is in case iii'.

Now

$$
\begin{aligned}
& K=\left\{\left(\begin{array}{cc}
A & 0 \\
0 & B
\end{array}\right) \in G: A \text { is a } p \times p \text { matrix and } B \text { is a } q \times q \text { matrix }\right\}, \\
& K_{1}=\left\{\left(\begin{array}{cc}
I & 0 \\
0 & B
\end{array}\right) \in G: B \text { is a } q \times q \text { matrix }\right\}, \\
& \mathfrak{g}=\left\{\left(\begin{array}{cc}
A & B \\
B^{*} & C
\end{array}\right): A \text { is a } p \times p \text { matrix, } C \text { is a } q \times q \text { matrix, } A=-A^{*}, C=-C^{*}\right\}, \\
& \mathfrak{p}=\left\{\left(\begin{array}{cc}
0 & B \\
B^{*} & 0
\end{array}\right): B \text { is a } p \times q \text { matrix }\right\}, \text { and } \\
& \mathfrak{a}=\left\{\left(\begin{array}{ccc}
0 & D & 0 \\
D & 0 & 0 \\
0 & 0 & 0
\end{array}\right): D \text { is a real } p \times p \text { diagonal matrix }\right\} .
\end{aligned}
$$


We may identify a with $\mathbf{R}^{p}$ as follows. If

$$
\left(\begin{array}{ccc}
0 & D & 0 \\
D & 0 & 0 \\
0 & 0 & 0
\end{array}\right) \in a,
$$

and $D=\sum_{i=1}^{p} x_{i} E_{i i}$, identify $D$ with the $p$-tuple, $\left(x_{1}, \ldots, x_{p}\right)$. In cases i, ii,

$$
\mathrm{a}^{+}=\left\{\left(x_{1}, \ldots, x_{p}\right): x_{1}>x_{2}>\cdots>x_{p}>0\right\} .
$$

In case iii' if $p<q$,

$$
\mathfrak{a}^{+}=\left\{\left(x_{1}, \ldots, x_{p}\right): x_{1}>x_{2}>\cdots>x_{p}>0\right\},
$$

while if $p=q$

$$
\mathfrak{a}^{+}=\left\{\left(x_{1}, \ldots, x_{p}\right): x_{1}>x_{2}>\cdots>x_{p-1}>\left|x_{p}\right|\right\} .
$$

Let $H_{r}=(1, \ldots, 1,0, \ldots, 0)$ with $r$ ones occurring. If $G$ is in case i, ii, or in case iii with $p<q$, any maximal parabolic subgroup of $G$ containing $B$ is $P\left(H_{r}\right)$ for some $r \leqslant p$. However, $K_{1} P\left(H_{r}\right) \neq G$ unless $r=p$. If $G=S O(p, p)$ let $E_{p}=$ $(1, \ldots, 1,-1)$. Then any maximal parabolic group $G$ containing $B$ is equal to $P\left(H_{r}\right)$ for $r \neq p-1$ or to $P\left(E_{p}\right)$. By direct calculations we now obtain the following result.

THEOREM 7.1. a. If $G=\operatorname{Sp}(p, q)$ with $2 \leqslant p \leqslant q$ and $K_{1}=\operatorname{Sp}(q), \quad \mathbf{P}\left(K_{1}\right)=$ $\left\{P\left(H_{0}\right)\right\}$.

b. If $G=S U(p, q)$ with $2 \leqslant p<q$ and $K_{1}=S U(q)$ or $U(q), \mathbf{P}\left(K_{1}\right)=\left\{P\left(H_{p}\right)\right\}$.

c. If $G=S U(p, p)$, and $K_{1}=S U(p), \mathbf{P}\left(K_{1}\right)=\varnothing$.

d. If $G=S U(p, p)$, and $K_{1}=U(p), \mathbf{P}\left(K_{1}\right)=\left\{P\left(H_{p}\right)\right\}$.

e. If $G=S O_{0}(p, q)$ with $3 \leqslant p<q$ and $K_{1}=S O(q), \mathbf{P}\left(K_{1}\right)=\left\{P\left(H_{p}\right)\right\}$.

f. If $G=S O(p, p)$ and $K_{1}=S O(p), \mathbf{P}\left(K_{1}\right)=\left\{P\left(H_{p}\right), P\left(E_{p}\right)\right\}$.

Remark. If $G=S O(4, q)$ and $S O(q) \subsetneq K_{1} \subsetneq K, K_{1} \cong S U(2) \times S O(q)$. In this case an elementary calculation shows that $\mathbf{P}\left(K_{1}\right)=\varnothing$.

8. Representations. Suppose $\Phi_{0}=\left\{\mu_{1}, \ldots, \mu_{l}\right\}$ and $\Phi=\left\{\alpha_{1}, \ldots, \alpha_{r}\right\}$. Fix $\left\{\lambda_{1}, \ldots, \lambda_{r}\right\} \subset \mathfrak{h}^{*}$ and $\left\{\gamma_{1}, \ldots, \gamma_{l}\right\} \subset$ a such that

$$
2\left\langle\lambda_{i}, \alpha_{j}\right\rangle /\left\langle\alpha_{j}, \alpha_{j}\right\rangle=\delta_{i j} \quad(i \leqslant i, j \leqslant r) ;
$$

and

$$
2\left\langle\gamma_{i}, \mu_{j}\right\rangle /\left\langle\mu_{j}, \mu_{j}\right\rangle=\delta_{i j} \quad(1 \leqslant i, j \leqslant l) .
$$

For $\Lambda \in \mathfrak{h}^{*}, \Lambda=\Lambda_{0}+\Lambda_{1}$ where $\Lambda_{1} \in(i \mathrm{t})^{*}$ and $\Lambda_{0} \in \mathfrak{a}^{*}$. For $I \subset\{1, \ldots, l\}$, and

$$
\Lambda_{0}=\sum_{i=1}^{l} a_{i} \gamma_{i}, \quad \text { set } \quad \Lambda_{0}(I)=\sum_{i \in I} a_{i} \gamma_{i}
$$

and $\Lambda(I)=\Lambda-\Lambda_{0}(I)$. Let $D$ be the set of all $\Lambda \in \mathfrak{h} *$ for which $2\left\langle\Lambda, \alpha_{j}\right\rangle /\left\langle\alpha_{j}, \alpha_{j}\right\rangle$ is a nonnegative integer for each $j \leqslant r$. Then $D$ is the set of dominant integral weights, and for $\Lambda \in D$ let $\pi_{\Lambda}: G \rightarrow G L\left(V_{\Lambda}\right)$ be the corresponding irreducible representation with highest weight $\Lambda$. Fix $v_{\Lambda}$ a nonzero highest weight vector of $V_{\Lambda}$. For $U \subset G$, define $D(U)=\left\{\Lambda \in D: v_{\Lambda} \in V_{\Lambda}^{U}\right\}$. Observe that $D(N)=D$. 
Proposition 8.2. Suppose $\left(K_{1}, \tilde{P}\right)$ is a reciprocity pair in $G$ and $\tilde{P}$ has Langlands decomposition $\tilde{M} \tilde{A} \tilde{N}$. Suppose $\Lambda \in D$, and $V_{\Lambda}^{K_{1}} \neq(0)$. Then, if $K_{1} \cap M=M_{1}$, $\Lambda \in D\left(M_{1}\right)$, and $\operatorname{dim} V_{\Lambda}^{K_{1}} \leqslant \operatorname{dim} V_{\Lambda}^{M_{1} \tilde{N}}$.

PROOF. If $\pi_{\Lambda^{*}}$ is the contragredient representation of $\pi_{\Lambda}, \operatorname{dim} V_{\Lambda}^{K_{1}}=\operatorname{dim} V_{\Lambda^{*}}^{K_{1}}$. Let (, , ) be the $G$-invariant bilinear pairing on $V_{\Lambda} \times V_{\Lambda^{*}}$. After suppressing $\pi_{\Lambda}$ and $\pi_{\Lambda^{*}}$, fix $u \in M_{1}, w \in V_{\Lambda^{*}}^{K_{1}}$ and consider the function $g \rightarrow\left(g u v_{\Lambda}, w\right)$. Now $g=k m a n$ with $k \in K_{1}, m \in \tilde{M}, a \in \tilde{A}$ and $n \in \tilde{N}$. Also $a v_{\Lambda}=\chi_{\Lambda}(a) v_{\Lambda}$ for all $a \in \tilde{A}$ where $\chi_{\Lambda}$ is a homomorphism of $\tilde{A}$ into $\mathbf{C} \sim\{0\}$. Then

$$
\begin{aligned}
\left(g u v_{\Lambda}, w\right) & =\left(k m a n u v_{\Lambda}, w\right)=\chi_{\Lambda}(a)\left(k^{\prime} m m^{-1} m v_{\Lambda}, w\right) \\
& =\chi_{\Lambda}(a)\left(m v_{\Lambda},\left(m u^{-1} m^{-1}\right) k^{-1} w\right)=\chi(a)\left(m v_{\Lambda}, w\right) .
\end{aligned}
$$

Hence, for all $g \in G,\left(u v_{\Lambda}-v_{\Lambda}, g^{-1} w\right)=0$. As $\pi_{\Lambda^{*}}$ is irreducible $u v_{\Lambda}-v_{\Lambda}=0$. Hence $\Lambda \in D\left(M_{1}\right)$. If $\operatorname{dim} V_{\Lambda}^{K_{1}}>\operatorname{dim} V_{\Lambda}^{M_{1} \tilde{N}}$, there is a nonzero $w \in V_{\Lambda}^{K_{1}}$ such that $(v, w)=0$, for all $v \in V_{\Lambda}^{M_{1} \tilde{N}}$. As $V_{\Lambda}^{M_{1} \tilde{N}}$ is $\tilde{P}$-invariant, $(g v, w) \equiv 0$. This contradicts the irreducibility of $\pi_{\Lambda}$.

Summarizing our results, we now describe $D\left(M_{1}\right)$ for all reciprocity pairs other than $(K, B)$. Fix $\Phi_{0}=\left\{\mu_{1}, \ldots, \mu_{l}\right\}, \Phi=\left\{\alpha_{1}, \ldots, \alpha_{r}\right\}$, and $\Lambda \in D$ such that $\Lambda=$ $\sum_{i=1}^{r} c_{i} \lambda_{i}$, where each $c_{i}$ is a nonnegative integer.

I. Suppose first that $\Phi_{0}=\left\{\mu_{1}, \mu_{2}, \mu_{3}, \mu_{4}\right\}$ has its root diagram

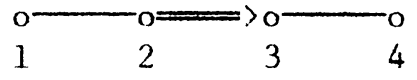

i. For $\left(G, K_{1}\right)=\left(F_{4(4)}, \operatorname{Sp}(3)\right), \Phi=\Phi_{0}$. Then $\Lambda \in D\left(M_{1}\right)$ if and only if $c_{3}$ and $c_{4}$ are even.

ii. For $\left(G, K_{1}\right)=\left(E_{6(2)}, S U(6)\right), \Phi=\left\{\alpha_{1}, \alpha_{2}, \alpha_{3}, \alpha_{4}, \alpha_{5}, \alpha_{6}\right\}$. Then, if $\alpha_{1}=\mu_{1}$, $\alpha_{2}=\mu_{2}, r\left(\alpha_{3}\right)=r\left(\alpha_{6}\right)=\mu_{4}$, and $r\left(\alpha_{4}\right)=r\left(\alpha_{5}\right)=\mu_{3}, \Lambda \in D\left(M_{1}\right)$ if and only if $c_{3}=c_{6}$ and $c_{4}=c_{5}$.

iii. For $\left(G, K_{1}\right)=\left(E_{7(-5)}, \operatorname{Spin}(12)\right), \Lambda \in D\left(M_{1}\right)$ if and only if $c_{i}=0$ whenever $\alpha_{i} \in \Phi_{00}$.

iv. For $\left(G, K_{1}\right)=\left(E_{8(-24)},\left(E_{7}\right)\right), \Lambda \in D\left(M_{1}\right)$ if and only if $c_{i}=0$ whenever $\alpha_{i} \in \Phi_{00}$.

II. Suppose $G / K$ is a Hermitian symmetric space of tube type, and $K_{1}=[K, K]$. Then $\Phi_{0}=\left\{\mu_{1}, \mu_{2}, \ldots, \mu_{l}\right\}$ has root diagram

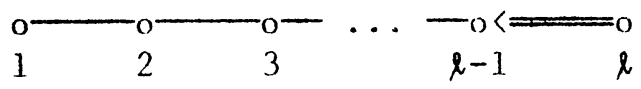

i. For $G=S U(l, l), \Lambda \in D\left(M_{1}\right)$ if and only if $c_{i}=c_{i^{\prime}}$, whenever $r\left(\alpha_{i}\right)=r\left(\alpha_{i^{\prime}}\right)$ $=\mu_{j}$, for $j<l$.

ii. For $G=\operatorname{Sp}(l, \mathbf{R}), \Phi=\Phi_{0}$. Then $\Lambda \in D\left(M_{1}\right)$ if and only if $c_{i}$ is even for all $i<l$. 
iii. For $G=\operatorname{Spin}_{0}(2, n)(l=2), \Lambda \in D\left(M_{1}\right)$ if and only if $c_{i}=0$ whenever $\alpha_{i} \in \Phi_{00}$.

iv. For $G=S O^{*}(4 l)\left(=\operatorname{Spin}_{0}^{*}(4 l)\right), \Lambda \in D\left(M_{1}\right)$ if and only if $c_{i}=0$ whenever $\alpha_{i} \in \Phi_{00}$.

v. For $G=E_{7(-25)}(l=3), \Lambda \in D\left(M_{1}\right)$ if and only if $c_{i}=0$ whenever $\alpha_{i} \in \Phi_{00}$.

III. Suppose $G / K$ is a Hermitian symmetric space of nontube type and $K_{1}=[K, K]$. Then $\Phi_{0}=\left\{\mu_{1}, \ldots, \mu_{l}\right\}$ has root diagrams

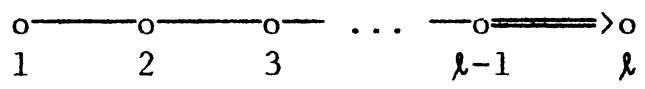

i. For $G=S U(l, k)$ with $l<k, \Lambda \in D\left(M_{1}\right)$ if and only if the following hold:

a. $c_{i}=c_{i^{\prime}}$ whenever $r\left(\alpha_{i}\right)=r\left(\alpha_{i^{\prime}}\right)=\mu_{j}$ for $j<l$; and,

b. $c_{i}=0$ whenever $\alpha_{i} \in \Phi_{00}$.

ii. For $G=S O^{*}(4 l+2), \Lambda \in D\left(M_{1}\right)$ if and only if $c_{i}=0$ whenever $\alpha_{i} \in \Phi_{00}$

iii. For $G=E_{6(-14)}, \Lambda \in D\left(M_{1}\right)$ if and only if $c_{i}=0$ whenever $\alpha_{i} \in \Phi_{00}$.

IV. Suppose now that $G$ is a Lorentz type group. It will be more convenient in these cases to consider Satake diagrams and take p for l as in $\S 7$.

i. For $\left(G, K_{1}\right)=(\operatorname{Sp}(p, q), \operatorname{Sp}(q))$ with $2 \leqslant p \leqslant q, \Phi=\left\{\alpha_{1}, \ldots, \alpha_{p+q}\right\}$ and $G$ has Satake diagram either

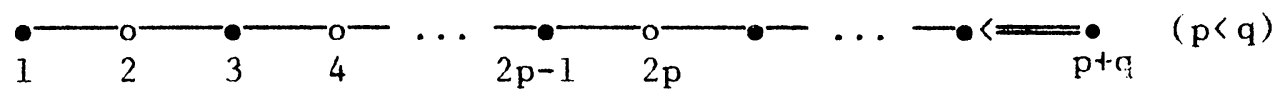

or

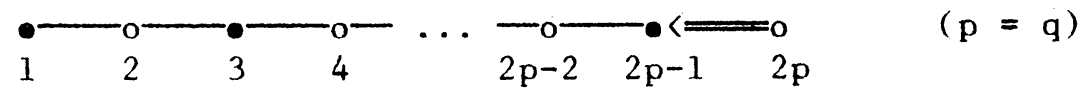

Then $\Lambda \in D\left(M_{1}\right)$ if and only if $c_{i}=0$ whenever $i>2 p$. This is a vacuous condition if $p=q$.

ii. For $\left(G, K_{1}\right)=(S U(p, q), S U(q))$ with $2 \leqslant p<q, \Lambda \in D\left(M_{1}\right)$ if and only if $c_{i}=0$ whenever $\alpha_{i} \in \Phi_{00}$.

iii. For $\left(G, K_{1}\right)=\left(\operatorname{Spin}_{0}(p, q)\right.$, Spin $\left.(q)\right)$ with $3 \leqslant p<q, \Lambda \in D\left(M_{1}\right)$ if and only if $c_{i}=0$ whenever $\alpha_{i} \in \Phi_{00}$.

iv. For $\left(G, K_{1}\right)=\left(\operatorname{Spin}_{0}(p, p), \operatorname{Spin}(p)\right), \Phi=\Phi_{0}=\left\{\alpha_{1}, \ldots, \alpha_{p}\right\}$ and $\Phi$ has root diagram

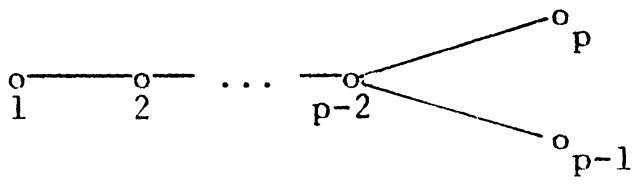

Then $\Lambda \in D\left(M_{1}\right)$ if and only if $c_{p-1}+c_{p}$ is even. In other words $\Lambda \in D\left(M_{1}\right)$ if and only if $\pi_{\Lambda}$ is a representation of $\mathrm{SO}_{0}(p, p)$. 
v. For $\left(G, K_{1}\right)=(S U(p, q), U(q))$ with $2 \leqslant p<q, \Phi=\left\{\alpha_{1}, \ldots, \alpha_{p+q-1}\right\}$, and $G$ has Satake diagram

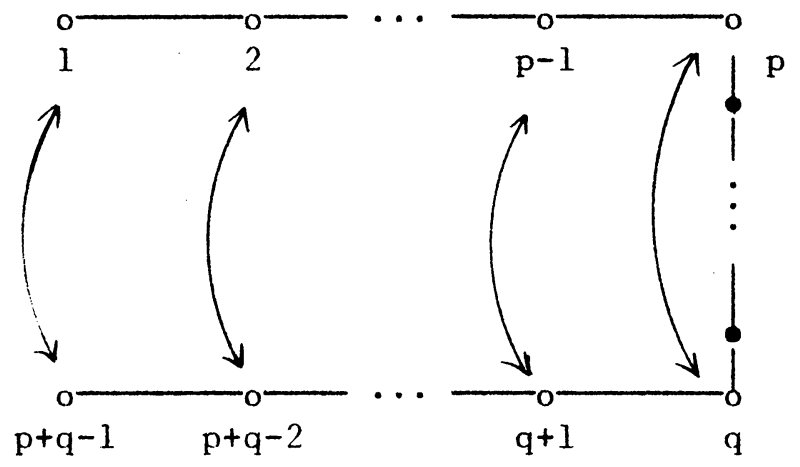

An elementary calculation shows that $\Lambda \in D\left(M_{1}\right)$ if and only if the following two conditions hold:

1. $c_{i}=0$ if $p<i<q$; and

2. $\sum_{j=1}^{p} j c_{j}=\sum_{j=0}^{p-1}(p-j) c_{q+j}$.

vi. For $\left(G, K_{1}\right)=(S U(p, p), U(p))$ with $2 \leqslant p, \Phi=\left\{\alpha_{1}, \ldots, \alpha_{2 p-1}\right\}$ and $G$ has Satake diagram

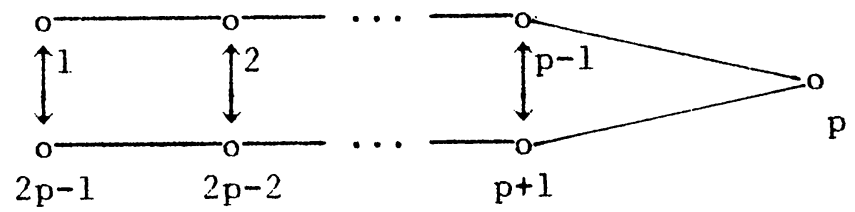

An elementary calculation shows that $\Lambda \in D\left(M_{1}\right)$ if and only if $\sum_{j=1}^{2 p-1} j c_{j} \in 2 p \mathbf{Z}$.

We shall say that $\left(G, K_{1}\right)$ is of type I, II, III or IV if $\left(G, K_{1}\right)$ is one of the above four types. To specify $\left(G, K_{1}\right)$ more precisely we shall also use lower case Roman numerals. The following classification will prove useful in $\S 9$.

Suppose $\left(G, K_{1}\right)$ is type I, II, or III, and $\Lambda \in \sum_{i=1}^{r} c_{i} \lambda_{i} \in D\left(M_{1}\right)$.

I. If $\left(G, K_{1}\right)$ is type I, $\Lambda \in D(M(\{3,4\}))$ if and only if $c_{1}=c_{2}=0$.

II. If $\left(G, K_{1}\right)$ is type II, $\Lambda \in D\left(M\left(\Phi_{0} \sim\left\{u_{l}\right\}\right)\right)$ if and only if $c_{p}=0$ whenever $\alpha_{p}=\mu_{l}$. (There is only one such $\alpha_{p}$.)

III. If $\left(G, K_{1}\right)$ is type III, $\Lambda \in D(M)$ if and only if $c_{p}=c_{p}$, whenever $r\left(\alpha_{p}\right)=$ $r\left(\alpha_{p^{\prime}}\right)=\mu_{l}$.

Identify $I \subset\{1, \ldots, l\}$ with the corresponding subset of $\Phi_{0}$. The following propositions follow immediately from the above classifications.

Proposition 8.3. Suppose $\left(G, K_{1}\right)$ is type I or II and $P(I) \in \mathbf{P}\left(K_{1}\right)$. For $\Lambda \in D\left(M_{1}\right)$ write $\Lambda=\Lambda(I)+\Lambda_{0}(I)$. Then $\Lambda(I) \in D\left(M_{1}\right)$ and $\Lambda_{0}(I) \in$ $D(M(I))$. 
Proposition 8.4. Suppose $\left(G, K_{1}\right)$ is type III, $r\left(\alpha_{p}\right)=r\left(\alpha_{p^{\prime}}\right)=\mu_{\text {l }}$ and $\Lambda=$ $\sum_{i=1}^{r} c_{i} \lambda_{i} \in D\left(M_{1}\right)$. Then, if $\Lambda_{0}=\sum_{i=1}^{r} d_{i} \lambda_{i}$ with $d_{i}=c_{i}$ for $i \notin\left\{p, p^{\prime}\right\}$ and $d_{p}=$ $d_{p^{\prime}}=\min \left\{c_{p}, c_{p^{\prime}}\right\}, \Lambda_{0} \in D(M)$. Moreover

$$
\Lambda=\Lambda-\Lambda_{0}=\left(c_{p}-d_{p}\right) \lambda_{p}+\left(c_{p^{\prime}}-d_{p}\right) \lambda_{p^{\prime}} \in D\left(M_{1}\right) \text {. }
$$

9. Our main result. The purpose of this section is to give a proof of Theorem $\mathrm{A}$.

Proposition 9.1. Suppose $\left(K_{1}, P(I)\right)$ is a reciprocity pair in $G$ and $\Lambda \in D(M(I))$. Then

$$
\operatorname{dim} V_{\Lambda}^{K_{1}}=\operatorname{dim} V_{\Lambda}^{M_{1} N(I)}=\operatorname{dim} V_{\Lambda}^{M(I) N(I)}=1 .
$$

Proof. This is an immediate corollary of Helgason's theorem and Proposition 8.2.

Recall that if $\Lambda=\Lambda_{1}+\Lambda_{2}$, with $\Lambda_{1}, \Lambda_{2} \in D$, the representation $\pi_{\Lambda}: G \rightarrow$ $G L\left(V_{\Lambda}\right)$ may be considered as a subrepresentation of $\pi_{\Lambda_{1}} \otimes \pi_{\Lambda_{2}}: G \rightarrow G L\left(V_{\Lambda_{1}} \otimes V_{\Lambda_{2}}\right)$ with $v_{\Lambda}=v_{\Lambda_{1}} \otimes v_{\Lambda_{2}}$.

LEMma 9.2. Suppose $\left(K_{1}, P(I)\right)$ is a reciprocity pair in $G$, and $\Lambda_{1}, \Lambda_{2} \in D\left(M_{1}\right)$ with $\operatorname{dim} V_{i}^{K_{1}}=\operatorname{dim} V_{i}^{M_{1} N(I)}$ where $V_{i}=V_{\Lambda_{i}}$. Then, if $\Lambda=\Lambda_{1}+\Lambda_{2}$,

$$
\operatorname{dim} V_{\Lambda}^{M_{1} N(I)}=\operatorname{dim} V_{\Lambda}^{K_{1}} .
$$

Proof. Set $\bar{V}_{i}=V_{i}^{M_{1} N(I)}, U_{i}=V_{i}^{K_{1}}$ and suppose $W_{i}$ is the $K_{1}$-invariant complement to $U_{i}$ in $V_{i}$. Then $V_{i}=U_{1} \oplus W_{i}$.

If $P_{i}: V_{i} \rightarrow U_{i}$, in the projection with kernel $W_{i}, P_{1} \oplus P_{2}: V_{1} \otimes V_{2} \rightarrow U_{1} \otimes U_{2}$, is the projection with kernel $W$ where

$$
W=V_{1} \otimes W_{2}+W_{1} \otimes V_{2}=V_{1} \otimes W_{2} \oplus W_{1} \otimes U_{2}
$$

As $P_{i}: \bar{V}_{i} \rightarrow U_{i}$ is an isomorphism, $P_{1} \otimes P_{2}: \bar{V}_{1} \otimes \bar{V}_{2} \rightarrow U_{1} \otimes U_{2}$ is an isomorphism. As $\bar{V}_{1} \otimes \bar{V}_{2}$ is $M(I)$-invariant $V_{\Lambda}^{M_{1} N(I)} \subset \bar{V}_{1} \otimes \bar{V}_{2}$. Hence $P_{1} \otimes P_{2}$ restricted to $V_{\Lambda}^{M_{1} N(I)}$ is an injection. Thus if $\tau \in V_{\Lambda}^{M_{1} N(I)}$,

$$
\tau=P_{1} \otimes P_{2}(\tau) \quad \bmod W
$$

Thus, as $W$ is $K_{1}$-invariant,

$$
\int_{K_{1}} \pi_{\Lambda}(k) \tau d k=P_{1} \otimes P_{2}(\tau) \quad \bmod W
$$

Thus $\operatorname{dim} V_{\Lambda}^{K_{1}} \geqslant \operatorname{dim} V_{\Lambda}^{M_{1} N(I)}$, and by Proposition 8.2 we have equality.

Definition 9.3. Suppose $\left(K_{1}, \tilde{P}\right)$ is a reciprocity pair in $G$ and $\Lambda \in D\left(M_{1}\right)$. We say that $\Lambda^{\prime}$ is p-related to $\Lambda$ if the following conditions hold:

(a) $V_{\Lambda^{\prime}} \subset \Lambda^{P} V_{\Lambda}$; and

(b) $v_{\Lambda^{\prime}} \in \Lambda^{p}\left(V_{\Lambda}^{M_{1} \tilde{N}}\right)$.

LemMa 9.4. Let $\left(K_{1}, \tilde{P}\right)$ be a reciprocity pair in $G$. Suppose $\Lambda, \Lambda^{\prime} \in D\left(M_{1}\right)$ and $\Lambda^{\prime}$ is p-related to $\Lambda$. Then, if

$$
\operatorname{dim} V_{\Lambda}^{K_{1}}=\operatorname{dim} V_{\Lambda}^{M_{1} \tilde{N}}, \quad \operatorname{dim} V_{\Lambda^{\prime}}^{K_{1}}=\operatorname{dim} V_{\Lambda^{\prime}}^{M_{1} \tilde{N}}
$$

Proof. Set $\bar{V}=V_{\Lambda}^{M_{1} \tilde{N}}, U=V_{\Lambda}^{K_{1}}$, and let $W$ be the $K_{1}$-invariant complement to $U$ in $V_{\Lambda}$. If $P: V_{\Lambda} \rightarrow U$ is the projection with kernel $W, \Lambda^{P} P: \Lambda^{P} V_{\Lambda} \rightarrow \Lambda^{P} U$ is the projection with kernel

$$
\bar{W}=\sum_{r=0}^{p-1} \Lambda^{r} U \otimes \Lambda^{p-r} W
$$


As $\Lambda^{P} P: \Lambda^{P} \bar{V} \rightarrow \Lambda^{P} U$ is an isomorphism, and $V_{\Lambda^{\prime}}^{M_{1} \tilde{N}} \subset \Lambda^{P} \bar{V}$, the restriction of $\Lambda^{P} P$ to $V_{\Lambda}^{M_{1} \tilde{N}}$ is injective. Hence, as in Lemma 9.2, we have our result.

Proposition 9.5. If $\left(G, K_{1}\right)$ is type I, II or III and $\mathbf{P}\left(K_{1}\right)=\{P(I)\}$, Theorem A holds.

Proof. 1. If $\left(G, K_{1}\right)$ is type I and $\Lambda \in D\left(M_{1}\right), \Lambda=\Lambda(\{3,4\})+\Lambda_{0}(\{3,4\})$. As $\Lambda\{3,4\}=c_{1} \lambda_{2}+c_{2} \lambda_{2}$, and $\lambda_{2}$ is 2-related to $\lambda_{1}$, it suffices to prove the result for $\Lambda=\lambda_{1}$. However in this case, $\pi_{\Lambda}$ is the adjoint representation, and hence

$$
\operatorname{dim} V_{\Lambda}^{M_{1} N(I)}=\operatorname{dim} V_{\Lambda}^{K_{1}}=3 .
$$

2. If $\left(G, K_{1}\right)$ is type II with $\Phi_{0}=\left\{\mu_{1}, \ldots, \mu_{l}\right\}$, suppose $\alpha_{p}=\mu_{l}$. From Proposition 8.3 and Lemma 9.2 is suffices to prove our result for $\Lambda=\lambda_{p}$. As $\operatorname{dim} V_{\Lambda}^{M_{1} N(I)}=2$, it suffices to show that $\operatorname{dim} V^{K_{1}}=2$. We now proceed case by case.

i. If $G=S U(l, l), V_{\Lambda}=\Lambda^{\prime} \mathbf{C}^{2 l}$ and $V_{\Lambda}^{K_{1}}$ is the span of $e_{1} \wedge \cdots \wedge e_{l}$ and $e_{1+1} \wedge \cdots \wedge e_{2 l}$ where $e_{1}, \ldots, e_{n}$ is the standard basis of $\mathbf{C}^{n}$.

ii. If $G=\operatorname{Sp}(l, \mathbf{R})$ with $\alpha_{l}=\mu_{l}, V_{\Lambda} \subset \Lambda^{\prime} \mathbf{C}^{2 l}$. Let $f_{j}=e_{j}-\sqrt{-1} e_{l+j}$ and $g_{j}=e_{j}+$ $\sqrt{-1} e_{l+j}$ for $j \leqslant l$. Then $V_{\Lambda}^{K_{1}}$ is the linear span of $f_{1} \wedge \cdots \wedge f_{l}$ and $g_{1} \wedge \cdots \wedge g_{l}$.

iii. If $G=\operatorname{Spin}_{0}(2, n) ; V_{\Lambda}=\mathbf{C}^{2+n}$ is the span of $e_{1}$ and $e_{2}$.

iv. If $G=S O^{*}(4 l), \pi_{\Lambda}$ is the half-spin representation on $\Lambda^{\text {even }} \mathbf{C}^{2 l}=V_{\Lambda}$. Hence $V_{\Lambda}^{K_{1}}=\Lambda^{0} \mathbf{C}^{2 l} \oplus \Lambda^{2 l} \mathbf{C}^{2 l}$.

v. If $G=E_{7(-25)}, \pi_{\Lambda}$ is a representation of degree 56 . As the only representations of $K_{1}=E_{6}$ of degree $\leqslant 56$ are of degree 1 and degree $27, \operatorname{dim} V_{\Lambda}^{K_{1}}=2$.

3. Suppose $\left(G, K_{1}\right)$ is type III. It suffices to prove our result for $\Lambda=\lambda_{p}$ or $\Lambda=\lambda_{p}^{\prime}$

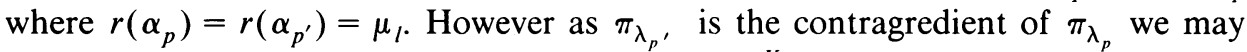
assume $\Lambda=\lambda_{p}$. It now suffices to prove that $\operatorname{dim} V_{\Lambda}^{K_{1}}=1$.

i. If $G=S U(l, k)$ with $l<k$, take $V_{\Lambda}=\Lambda^{\prime} \mathbf{C}^{l+k}$. Then $V_{\Lambda}^{K_{1}}=\mathbf{C} e_{1} \wedge \cdots \wedge e_{\text {l. }}$.

ii. If $G=S O^{*}(4 l+2)$ take $V_{\Lambda}=\Lambda^{\text {even }} \mathbf{C}^{2 l+1}$. Then $V_{\Lambda}^{K_{1}}=\mathbf{C}$.

iii. If $G=E_{6(-14)}, \operatorname{dim} V_{\Lambda}=27$. As the only irreducible representations of $K_{1}=$ $\operatorname{Spin}(12)$ of degree $\leqslant 27$ are of degree 1,10 , and 16 we must have $\operatorname{dim} V_{\Lambda}^{K_{1}}=1$.

Proposition 9.6. Suppose $\left(G, K_{1}\right)$ is type IV, and $\left(G, K_{1}\right) \neq\left(\operatorname{Spin}_{0}(n, n)\right.$, $\operatorname{Spin}(n))$ or $\left(G, K_{1}\right) \neq(S U(n, n), U(n))$ for any $n$. Then Theorem A holds.

Proof. 1. Suppose $\left(G, K_{1}\right)=(\operatorname{Sp}(p, q), \operatorname{Sp}(q))$. Then $\Phi_{0}=\left\{\mu_{1}, \ldots, \mu_{p}\right\}$ and $\left\{P\left\{\mu_{p}\right\}\right\}=\mathbf{P}\left(K_{1}\right)$. Using our notation of $\S 8$ and applying Lemmas 9.2 and 9.4 it suffices to prove our result for $\Lambda=\lambda_{1}$. In this case $\pi_{\Lambda}$ is the standard representation on $\mathrm{C}^{2(p+q)}$. Now $M(I) / M_{1}=\mathrm{Sp}(p, \mathbf{C})$, and so

$$
\operatorname{dim} V_{\Lambda}^{K_{1}}=\operatorname{dim} V_{\Lambda}^{M_{1} N(I)}=2 p .
$$

2. If $\left(G, K_{1}\right)=\left(\operatorname{Spin}_{0}(p, q), \operatorname{Spin}(q)\right)$ with $p<q, M(I) / M_{1} \cong S L(p, \mathbf{R})$. By Lemmas 9.2 and 9.4 it suffices to prove our result for $\pi_{\Lambda}$ the standard representation. In this case,

$$
\operatorname{dim} V_{\Lambda}^{K_{1}}=\operatorname{dim} V_{\Lambda}^{M_{1} N(I)}=p .
$$

3. If $\left(G, K_{1}\right)=(S U(p, q), S U(q))$ with $p<q, M(I) / M_{1} \cong S L(p, \mathrm{C})$, and by Lemmas 9.2 and 9.4 it suffices to prove our result for $\pi_{\Lambda}$ the standard representations on $\mathbf{C}^{p+q}$ or for $\pi_{\Lambda}$ the representation on $\Lambda^{p+q-1} \mathbf{C}^{p+q}$. As these representations are contragredient it suffices to assume that $\pi_{\Lambda}$ is the standard representation. 
In this case,

$$
\operatorname{dim} V_{\Lambda}^{K_{1}}=\operatorname{dim} V_{\Lambda}^{M_{1} N(I)}=p .
$$

4. Suppose $\left(G, K_{1}\right)=(S U(p, q), U(q))$ with $p<q$. Let $\Lambda=\sum_{j=1}^{p+q-1} c_{j} \lambda_{j}$. Then $c_{j}=0$ if $p<j<q$, and

$$
\sum_{j=1}^{p} c_{j} j+\sum_{j=0}^{p-1}(j-p) c_{q+j}=0 .
$$

Now $\pi_{\Lambda}$ is a subrepresentation on the space $S^{c_{1}}\left(\mathbf{C}^{p+q}\right) \otimes \cdots \otimes S^{c_{p}}\left(\Lambda^{p} \mathbf{C}^{p+q}\right) \otimes S^{c_{q}}\left(\Lambda^{q} \mathbf{C}^{p+q}\right) \otimes \cdots \otimes S^{c_{p+q-1}}\left(\Lambda^{p+q-1} \mathbf{C}^{p+q}\right)$. Let $w_{1}=e^{i q \theta}$ and $w_{2}=e^{-i p \theta}$ and

$$
z=\left(\begin{array}{cc}
w_{1} I_{p} & 0 \\
0 & w_{2} I_{q}
\end{array}\right)
$$

Then on $V_{\Lambda}^{\left[K_{1}, K_{1}\right]}$

$$
\begin{aligned}
\pi(z) & =w_{1}^{\left(\sum_{j=1}^{p} j c_{j}+\sum_{j=0}^{r_{j}-1} j c_{j+q}\right)} w_{2}^{\left(q \sum_{j=0}^{p-1} c_{j+q}\right)} \\
& =w_{1}^{\left(p \sum_{j=0}^{p-1} c_{j+q}\right)} w_{2}^{\left(q \sum_{j=0}^{p-1} c_{j+q}\right)}=1 .
\end{aligned}
$$

Hence by 3 ,

$$
\operatorname{dim} V_{\Lambda}^{K_{1}}=\operatorname{dim} V_{\Lambda}^{M_{1} N(I)}
$$

Proposition 9.7. Suppose $\left(G, K_{1}\right)=\left(\operatorname{Spin}_{0}(n, n), \operatorname{Spin}(n)\right)$ for some $n \geqslant 3$. Then Theorem A holds.

Proof. This is the only case where $\left|\mathbf{P}\left(K_{1}\right)\right|=2$. Then $M_{1}=\mathbf{Z}_{2}$ and $M(I) / M_{1}=$ $S L(n, \mathbf{R})$ for both $I$. Now $\Phi=\Phi_{0}=\left\{\alpha_{1}, \ldots, \alpha_{n}\right\}$ has root diagram

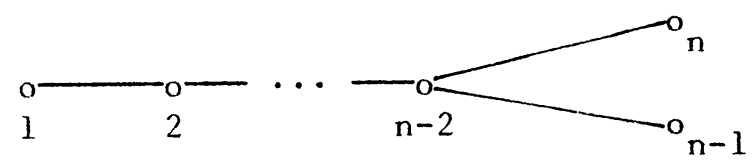

From Lemmas 9.2 and 9.4, it suffices to prove our result for $\Lambda=\lambda_{1}, \Lambda=\lambda_{n-1}+\lambda_{n}$, and $\Lambda=2 \lambda_{n-1}$ or $\Lambda=2 \lambda_{n}$.

Now $\mathbf{P}\left(K_{1}\right)=\left\{P\left\{\alpha_{n-1}\right\}, P\left\{\alpha_{n}\right\}\right\}$.

1. Clearly, $\operatorname{dim} V_{\lambda_{1}}^{K_{1}}=\operatorname{dim} V_{\lambda_{1}}^{M_{1} N(I)}=n$ for either $I$.

2. Now $\Lambda^{n} \mathbf{C}^{2 n}=V_{2 \lambda_{n-1}} \oplus V_{2 \lambda_{n}}$. As $\left(\Lambda^{n} \mathbf{C}^{2 n}\right)^{K_{1}}$ is the span of $e_{1} \wedge \cdots \wedge e_{n}$ and $e_{n+1} \wedge \cdots \wedge e_{2 n}$, and as

$$
\min \operatorname{dim}\left\{V_{\Lambda}^{M_{1} N(I)}: P(I) \in \mathbf{P}\left(K_{1}\right)\right\}=1
$$

for $\Lambda=2 \lambda_{n-1}$ or $2 \lambda_{n}$ we are done.

3. If $\Lambda=\lambda_{n-1}+\lambda_{n}, \Lambda^{n-1} \mathbf{C}^{2 n}=V_{\Lambda}$. Then

$$
\operatorname{dim} V_{\Lambda}^{K_{1}}=n=\operatorname{dim} V_{\Lambda}^{M_{1} M(I)}=\operatorname{dim} V_{\Lambda}^{M_{1} N\left(I^{\prime}\right)}
$$

where $I=\left\{\alpha_{n+1}\right\}$ and $I^{\prime}=\left\{\alpha_{n}\right\}$. 
EXAMPLE. It remains only to consider the case where $G=S U(n, n)$. This group has Satake diagram

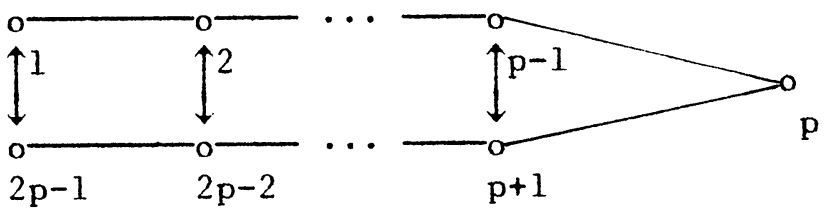

Let $K_{1}=S U(n)$ and consider $I=\left\{\alpha_{p}\right\}$. Although $K_{1} P(I) \neq G$ we have that $K_{1} \cap P(I)$ is normal in the reductive part of $P(I)$. For any $\Lambda \in D$, we wish to show that

$$
\operatorname{dim} V_{\Lambda}^{K_{1}}=\operatorname{dim} V_{\Lambda}^{M_{1} M(I)}
$$

Modifying the statements of Lemmas 9.2 and 9.4 it suffices to prove this equality for $\Lambda=\lambda_{1}$. However, as this is the standard representation, the equality is clear.

Finally from the above example we now obtain the following result.

Proposition 9.9. Suppose $\left(G, K_{1}\right)=(S U(n, n), U(n))$, and $\Lambda=\sum_{j=1}^{2 p-1} c_{j} \lambda_{j} \in D$. Then $V_{\Lambda}^{K_{1}} \neq(0)$ if and only if

$$
\sum_{j=1}^{p-1} j c_{j}=\sum_{j=0}^{p}(p-j) c_{p+j},
$$

and in this case

$$
\operatorname{dim} V_{\Lambda}^{K_{1}}=\operatorname{dim} V_{\Lambda}^{M_{1} N(I)}
$$

where $P(I) \in \mathbf{P}\left(K_{1}\right)$.

This completes the proof of Theorem A.

REMARK. The case $\left(G, K_{1}\right)=(S U(n, n), U(n))$ is the only case where $\Lambda \in D\left(M_{1}\right)$ does not guarantee $V_{\Lambda}^{K_{1}} \neq(0)$.

\section{REFERENCES}

[A] S. Araki, On root systems and an infinitesimal classification of irreducible symmetric spaces, J. Math. Osaka City Univ. 13 (1962), 1-34.

[G] S. Gelbart, $A$ theory of Stiefel harmonics, Trans. Amer. Math. Soc. 192 (1974), 29-50.

[G-K] K. Gross and R. Kunze, Finite dimensional induction and new results on invariants for classical groups. I, Amer. J. Math. 106 (1984), 893-974.

[H1] S. Helgason, Differential geometry and symmetric spaces, Academic Press, New York, 1962.

[H2] _ A duality for symmetric spaces with applications to group representations, Adv. in Math. 5 (1970), 1-154.

[J] K. Johnson, Generalized Hua operator and parabolic subgroups, Ann. of Math. 120 (1984), 477-495.

[K-W] A. Koranyi and J. A. Wolf, Realization of Hermitian symmetric spaces as generalized half-planes, Ann. of Math. 81 (1965), 265-288.

[K-R] B. Kostant and S. Rallis, Orbits and representations associated with symmetric spaces, Amer. J. Math. 93 (1971), 753-809.

[L] O. Loos, Symmetric Spaces, II; Compact spaces and classification, Benjamin, New York, 1969.

[M] C. C. Moore, Compactifications of symmetric spaces, II; The Cartan domain, Amer. J. Math. 86 (1964), 358-378.

[O] A. L. Onischik, Inclusion relations among transitive compact transformation groups, Amer. Math. Soc. Transl. (2) 50 (1966), 5-58. 
[S] H. Schlichtkrull, One-dimensional K-types in finite dimensional representations of semisimple Lie groups: A generalization of Helgason's theorem, Math. Scand. 54 (1984), 279-294.

[St] R. Steinberg, Lectures on Chevalley groups, Yale Univ., New Haven, Conn., 1967.

[W] N. R. Wallach, Harmonic analysis on homogeneous spaces, Dekker, New York, 1973.

[We] H. Weyl, The classical groups, Princeton Univ. Press, Princeton, N. J., 1946.

Department of Mathematics, University of Georgia, Athens, Georgia 30602 